Pacific Journal of Mathematics

DEVELOPMENT OF THE MAPPING FUNCTION AT A 


\title{
DEVELOPMENT OF THE MAPPING FUNCTION AT A CORNER
}

\author{
Neil M. Wigley
}

Let $D$ be a domain in the plane which is partially bounded by two curves $\Gamma_{1}$. and $\Gamma_{2}$ which meet at the origin and form there an interior angle $\pi \tau>0$. Let $N$ be an integer $\geqq 2$ and let $\alpha$ be a real number such that $0<\alpha<1$. Suppose that for $i=1,2, \Gamma_{i}$ admits a parametrization $x=x_{i}(t), y=y_{i}(t), 0 \leqq t \leqq 1$, where $x_{i}$ and $y_{i}$ have $N$ th derivatives which are uniformly $\alpha$ Hölder continuous, and $\left|x_{i}^{\prime}(t)\right|+\left|y_{i}^{\prime}(t)\right|>0$. Let $F(z)$ map the upper half plane conformally onto $D$ in such a way that $F(0)=$ 0 . Then if $\tau$ is irrational $F(z)$ has an asymptotic expansion in powers of $z$ and $z^{\tau}$, with error term $o\left(z^{N \tau-\varepsilon}\right)$. If $\tau=p / q$, a reduced fraction, then $F(z)$ has an asymptotic expansion in powers of $z, z^{\tau}$, and $z^{p} \log z$, with error term $o\left(z^{N \tau-\varepsilon}\right)$. In both cases $\varepsilon$ is an arbitrarily small positive number. Furthermore expansions for derivatives of $F(z)$ of order $\leqq N$ may be obtained by differentiating formally.

The behavior of such conformal maps at corners was first investigated by Lichtenstein [9]. Let $F^{-1}(z)$ be the function inverse to $F(z)$ which maps $D$ onto the upper half plane. Lichtenstein showed that if $\Gamma_{1}$ and $\Gamma_{2}$ are analytic then

$$
\frac{d}{d z} F^{-1}(z)=z^{1 / \tau-1} \varphi(z)
$$

where $\varphi(z)$ is continuous in $\bar{D}$ and $\varphi(0) \neq 0$. This result was later generalized in two ways. One was to weaken the requirements on $\Gamma_{1}$ and $\Gamma_{2}$. It follows from the work of Kellogg [4] and Warschawski [10] that with very modest conditions imposed on $\Gamma_{1}$ and $\Gamma_{2}$ one has

$$
F^{-1}(z)=z^{3 / \tau} \varphi(z)
$$

where again $\varphi(z)$ is continuous in $\bar{D}$ and $\varphi(0) \neq 0$. In particular this follows if one assumes that $\Gamma_{1}$ and $\Gamma_{2}$ have continuously turning tangents in a neighborhood of the origin (though weaker conditions will suffice).

The other generalization of Lichtenstein's theorem was an improvement of the result (1.1), maintaining the analyticity requirement. For the case $\tau=1$ Lewy [8] showed that $F(z)$ has an asymptotic expansion

Received August 9, 1964. This work was performed under the auspices of the U.S. Atomic Energy Commission. The author wishes to thank Professor R. Sherman Lehman for suggesting this problem. 
in powers of $z$ and $\log z$. Later Lehman [6] showed that expansions of the kind mentioned in the first paragraph are valid for all angles $\pi \tau>0$, provided $\Gamma_{1}$ and $\Gamma_{2}$ are analytic. Thus in this paper we dovetail the results of the two developments. Furthermore we shall indicate some applications to the behavior at corners of solutions of elliptic partial differential equations; see [3], [5], [7], [8], [11] and [12].

2. Principal results. Let $N$ be an integer $\geqq 2$ and let $\alpha$ be a real number such that $0<\alpha<1$. Assume that for $i=1,2, \Gamma_{i}$ admits a parametrization $x=x_{i}(t), y=y_{i}(t)$ where $x_{i}(t)$ and $y_{i}(t)$ are uniformly $C^{N+\alpha}$ for $0 \leqq t \leqq 1,{ }^{1}$ and assume that there exists a $\delta>0$ such that $\left|x_{i}^{\prime}(t)\right|+\left|y_{i}^{\prime}(t)\right| \geqq \delta$ for $0 \leqq t \leqq 1$. Let $F(z)$ map the upper half plane conformally onto $D$. Then $G(z) \equiv F\left(z^{1 / \tau}\right)$ maps the sector $0<\arg z<\pi \tau$ onto $D$ and we have the following theorems.

THEOREM 1. If $\tau$ is irrational then there exists a polynomial $P\left(z, z^{\tau}\right)$ such that as $z \rightarrow 0,0 \leqq \arg z \leqq \pi$,

$$
F(z)=z^{\tau} P\left(z, z^{\tau}\right)+o\left(z^{N \tau-\varepsilon}\right)
$$

where $\varepsilon$ is an arbitrarily small positive number and $P(0,0) \neq 0$. If $\tau=$ $p / q$, a reduced fraction, then there exists a polynomial $P\left(z, z^{\tau}, z^{p} \log z\right)$ such that as $z \rightarrow 0,0 \leqq \arg z \leqq \pi$,

$$
F(z)=z^{\tau} P\left(z, z^{\tau}, z^{p} \log z\right)+o\left(z^{N \tau-\varepsilon}\right)
$$

where $\varepsilon$ is an arbitrarily small positive number and $P(0,0,0) \neq 0$. Furthermore expansions for derivatives of order $\leqq N$ may be obtained by differentiating formally.

THEOREM 2. If $\tau$ is irrational then there exists a polynomial $P\left(z, z^{1 / \tau}\right)$ such that as $z \rightarrow 0,0 \leqq \arg z \leqq \pi \tau$,

$$
G(z)=z P\left(z, z^{1 / \tau}\right)+o\left(z^{N-\varepsilon}\right)
$$

where $\varepsilon$ is an arbitrarily small positive number and $P(0,0) \neq 0$. If $\tau=$ $p / q$, a reduced fraction, then there exists a polynomial $P\left(z, z^{1 / \tau}, z^{q} \log z\right)$ such that as $z \rightarrow 0,0 \leqq \arg z \leqq \pi \tau$,

$$
G(z)=z P\left(z, z^{1 / \tau}, z^{q} \log z\right)+o\left(z^{N-\varepsilon}\right)
$$

where $\varepsilon$ is an arbitrarily small positive number and $P(0,0,0) \neq 0$. Furthermore expansions for derivatives of order $\leqq N$ may be obtained by differentiating formally.

${ }^{1}$ This means there exists a constant $K$ such that for $0 \leqq s<t \leqq 1$ and $0 \leqq n \leqq N$

$$
\left|\frac{d^{n}}{d t^{n}} x_{i}(s)-\frac{d^{n}}{d t^{n}} x_{i}(t)\right|+\left|\frac{d^{n}}{d t^{n}} y_{i}(s)-\frac{d^{n}}{d t^{n}} y_{i}(t)\right| \leqq K|s-t|^{\infty}
$$


From Theorems 1 and 2 one can obtain an asymptotic expansion for the inverse function $F^{-1}(z)$ which maps $D$ onto the upper half plane. The method is an iterative one, starting with $F(z)=o\left(z^{\tau-\varepsilon}\right)$ and increasing the exponent of the error term; see, for instance, Wasow [11], pp. 49-50.

THEOREM 3. If $\tau$ is irrational then there exists a polynomial $P\left(z, z^{1 / \tau}\right)$ such that as $z \rightarrow 0, z \in D \cup \Gamma_{1} \cup \Gamma_{2}$,

$$
F^{-1}(z)=z^{1 / \tau} P\left(z, z^{1 / \tau}\right)+o\left(z^{N-1+1 / \tau-\varepsilon}\right)
$$

where $\varepsilon$ is an arbitrarily small positive number and $P(0,0) \neq 0$. If $\tau=$ $p / q$, a reduced fraction, then there exists a polynomial $P\left(z, z^{1 / \tau}, z^{q} \log z\right)$ such that as $z \rightarrow 0, z \in D \cup \Gamma_{1} \cup \Gamma_{2}$,

$$
F^{-1}(z)=z^{1 / \tau} P\left(z, z^{1 / \tau}, z^{q} \log z\right)+o\left(z^{N-1+1 / \tau-\varepsilon}\right)
$$

where $\varepsilon$ is an arbitrarily small positive number and $P(0,0,0) \neq 0$. Furthermore expansions for derivatives of order $\leqq N$ may be obtained by differentiating formally,

Since $G^{-1}(z)=(F(z))^{\tau}$, we have, by the binomial theorem.

THEOREM 4. If $\tau$ is irrational there exists a polynomial $P\left(z, z^{1 / \tau}\right)$ such that as $z \rightarrow 0, z \in D \cup \Gamma_{1} \cup \Gamma_{2}$,

$$
G^{-1}(z)=z P\left(z, z^{1 / \tau}\right)+o\left(z^{N-\varepsilon}\right)
$$

where $\varepsilon$ is an arbitrarily small positive number and $P(0,0) \neq 0$. If $\tau=$ $p / q$, a reduced fraction, then there exists a polynomial $P\left(z, z^{1 / \tau}, z^{q} \log z\right)$ such that as $z \rightarrow 0, z \in D \cup \Gamma_{1} \cup \Gamma_{2}$,

$$
G^{-1}(z)=z P\left(z, z^{1 / \tau}, z^{q} \log z\right)+o\left(z^{N-\varepsilon}\right)
$$

where $\varepsilon$ is an arbitrarily small positive number and $P(0,0,0) \neq 0$. Furthermore expansions for derivatives of order $\leqq N$ may be obtained by differentiating formally.

3. Applications to partial differential equations. The expansions of Theorems 2 and 4 have immediate applications to a previous paper of the author [12]. In particular $\S 4$ and 5 of [12] need only be modified suitably to obtain the following theorems.

Let $U(x, y)$ be a solution in $D$ of the partial differential equation

$$
\frac{\partial^{2} U}{\partial x^{2}}+\frac{\partial^{2} U}{\partial y^{2}}+K U=F
$$

where $K$ and $F$ are $(N-1)$-times continuously differentiable in $D \cup \Gamma_{1} \cup \Gamma_{2} \cup\{0\}, U$ is twice continuously differentiable in $D$, and $U_{x}$ 
and $U_{y}$ are $\alpha$-Hölder continuous in every compact subset of $D \cup \Gamma_{1} \cup \Gamma_{2}$. We also assume that for $i=1,2, U$ satisfies on $\Gamma_{i}$ a boundary condition

$$
\delta_{i} \frac{\partial U}{\partial n}+A_{i} U=B_{i}
$$

where $\delta_{i}=0$ or $1, \partial / \partial n$ represents the outgoing normal derivative, and $A_{i}$ and $B_{i}$ are $(N-1)$-times continuously differentiable as functions of arc length, defined on $\Gamma_{i} \cup\{0\}$, and $A_{i}(0) \neq 0$, if $\delta_{i}=0$. Finally, we assume that as $z \rightarrow 0, z \in D \cup \Gamma_{1} \cup \Gamma_{2}$,

$$
U(z)=o\left(z^{\mu}\right)
$$

where $\mu>\max (-1,-1 / \tau)$ if $\delta_{1}=\delta_{2}$ and $\mu>\max (-1,-1 / 2 \tau)$ if $\delta_{1} \neq \delta_{2}$. Then

Theorem 5. If $\delta_{1}=\delta_{2}=0$ then as $z \rightarrow 0, z \in D \cup \Gamma_{1} \cup \Gamma_{2}$,

$$
U(z)=\log z P_{1}+\log \bar{z} P_{2}+P_{3}+o\left(z^{N-1-\bar{\varepsilon}}\right)
$$

where $P_{1}, P_{2}$ and $P_{3}$ are polynomials in $z, \bar{z}, z^{1 / \tau}$ and $\bar{z}^{1 / \tau}$ if $\tau$ is irrational and in $z, \bar{z}, z^{1 / \tau}, \bar{z}^{1 / \tau}, z^{q} \log z$ and $\bar{z}^{q} \log \bar{z}$ if $\tau=p / q$; and $\varepsilon$ is an arbitrarily small positive number. If $B_{1}(0) A_{2}(0)=B_{2}(0) A_{1}(0), P_{1}$ and $P_{2}$ vanish identically. Furthermore expansions for derivatives of $U(z)$ of order $\leqq N-2$ may be obtained by differentiating formally.

THEOREM 6. If $\delta_{1}=0$ and $\delta_{2}=1$ (or $\delta_{1}=1$ and $\left.\delta_{2}=0\right)$ then as $z \rightarrow 0, z \in D \cup \Gamma_{1} \cup \Gamma_{2}$,

$$
U(z)=P+o\left(z^{N-1-\varepsilon}\right)
$$

where $P$ is a polynomial in $z, \bar{z}, z^{1 / 2 \tau}$ and $\bar{z}^{1 / 2 \tau}$ if $\tau$ is irrational; $P$ is a polynomial in $z, \bar{z}, z^{1 / 2 \tau}, \bar{z}^{1 / 2 \tau}, z^{q} \log z$ and $\bar{z}^{q} \log \bar{z}$ if $\tau=p / q$ and $q$ is odd; $P$ is a polynomial in $z, \bar{z}, z^{1 / 2 \tau}, \bar{z}^{1 / 2 \tau}, z^{q / 2} \log z$ and $\bar{z}^{q / 2} \log \bar{z}$ if $\alpha=p / q$ and $q$ is even; and $\varepsilon$ is an arbitrarily small positive number. Furthermore expansions for derivatives of $U(z)$ of order $\leqq N-2$ may be obtained by differentiating formally.

TheOREM 7. If $\delta_{1}=\delta_{2}=1$ then as $z \rightarrow 0, z \in D \cup \Gamma_{1} \cup \Gamma_{2}$,

$$
U(z)=\log z P_{1}+\log \bar{z} P_{2}+P_{3}+o\left(z^{N-\delta}\right)
$$

where $P_{1}, P_{2}$ and $P_{3}$ are polynomials in $z, \bar{z}, z^{1 / \tau}$ and $\bar{z}^{1 / \tau}$ if $\tau$ is irrational and in $z, \bar{z}, z^{1 / \tau}, \bar{z}^{1 / \tau}, z^{q} \log z$ and $\bar{z}^{q} \log \bar{z}$ if $\tau=p / q$; and $\varepsilon$ is an arbitrarily small positive number. If $U(z)$ is bounded at the origin then $P_{1}$ and $P_{2}$ vanish identically. Furthermore derivatives of $U(z)$ of order $\leqq N-1$ may be obtained by differentiating formally. 
4. Some Lemmas. Later we shall need some properties of functions which are Hölder continuous in a set, but whose Hölder constants diverge in a certain way near a boundary point of the set. Let $S$ be a subset of the plane which does not contain the origin, but of which the origin is a cluster point. Let $\mu$ and $\beta$ be real numbers, $0 \leqq \beta<1$, and let $M$ be a nonnegative integer. Let $f(x, y)$ be a real or complex valued function such that $f(x, y) \in C^{\mu+\beta}$ for $(x, y) \in S$, and suppose that for $0 \leqq n \leqq M$

(i) $D^{n} f(x, y)=O\left(z^{\mu-n}\right)$

as $z \rightarrow 0, z \in S$, where $D^{n}$ ranges over all $n$th order partial derivatives, and

(ii) there exists a constant $K$ such that

$$
\sup \frac{\left|D^{n} f(z)-D^{n} f(\zeta)\right|}{|z-\zeta|^{\beta}}|z|^{n+\beta-\mu} \leqq K
$$

where the supremum is taken over all derivatives $D^{n}$, and over all points $z, \zeta \in S$ such that $0<|z-\zeta|<\delta|z|, \delta|\zeta| ; \delta$ is assumed to be some positive number $<1$. The totality of such functions we designate by $W_{\mu}^{M+\beta}(S)$. If $S$ is the sector $\delta_{1} \leqq \arg z \leqq \delta_{2}, 0<|z|<\left|z_{0}\right|$, we write $W_{\mu}^{M+\beta}\left(\left[\delta_{1}, \delta_{2}\right]\right)$. We omit the dependence on $z_{0}$ because we are only concerned with properties (i) and (ii) in some neighborhood of the origin. If $S$ is a segment $0<x<A$ we write $W_{\mu}^{M+\beta}$; properties (i) and (ii) should then be modified properly for a function of one variable. We observe that if $\beta=0$ property (ii) follows from property (i) and the condition $|z-\zeta|<\delta|z|, \delta|\zeta|$ 。

We now list some properties of the $W$-spaces. We state them for the complex case, though with suitable modifications the properties hold for the real case. Thus we assume $0<|z-\zeta|<\delta|z|, \delta|\zeta|$, and $z, \zeta \in S$.

1. $1-\hat{o}<|z / \zeta|<1+\delta$.

2. Let $\mu \leqq-1$. If $(\partial / \partial x) f(z),(\partial / \partial y) f(z) \in W_{\mu}^{0}(S)$ then $f(z)$ differs by a constant from a function in $W_{\mu+1}^{1}(S)$. The proof is contained in Bourbaki [2].

3. If $(\partial / \partial x) f(z),(\partial / \partial y) f(z) \in W_{\mu}^{0}(S)$ then $f$ differs by a constant from a function in $W_{\mu+1}^{\beta}(S), 0 \leqq \beta<1$. The proof follows from property 2 above and the mean value theorem for functions of two variables.

4. There exists a constant $K$ depending only on $\mu, \beta$ and $\delta$ such that

$$
\left|z^{\mu+\beta}-\zeta^{\mu+\beta}\right| \leqq K|z|^{\mu}\left|z^{\beta}-\zeta^{\beta}\right| \leqq K|z|^{\mu}|z-\zeta|^{\beta} .
$$

Here we assume that $S$ is so chosen that $z^{\mu+\beta}$ and $z^{\beta}$ are single valued functions.

5. Let $f(z) \equiv z^{\mu}$, and assume $z^{\mu}$ is single valued for $z \in S$. Then 
for all integers $M \geqq 0$ and any $\beta$ such that $0 \leqq \beta \leqq 1, f(z) \in W_{\mu}^{M+\beta}(S)$.

6. Let $M$ and $N$ be integers $\geqq 0$, let $\alpha$ and $\beta$ satisfy $0<\alpha, \beta<1$, and let $\mu$ and $\nu$ be real numbers. Let $f(z) \in W_{\mu}^{M+\alpha}(S)$ and $g(z) \in W_{\nu}^{N+\beta}(S)$. Let $\alpha^{\prime}=\min (\alpha, \beta), M^{\prime}=\min (M, N)$ and $\mu^{\prime}=\min (\mu, \nu)$. Then

$$
f(z)+g(z) \in W_{\mu \mu^{\prime}}^{M \alpha^{\prime}+\alpha^{\prime}}(S)
$$

and

$$
f(z) g(z) \in W_{\mu \nu}^{M^{\prime}+\alpha^{\prime}}(S)
$$

Proof. The first statement follows from the fact that $W_{\mu^{\prime}}^{M \alpha^{\prime}+\alpha^{\prime}}(S) \supseteqq$ $W_{\mu}^{M+\alpha}(S) \cap W_{\nu}^{N+\beta}(S)$, and because the $W$-spaces are linear. For the second statement we observe first that $f(z) g(z) \in C^{M^{\prime}+\alpha^{\prime}}(S)$. Then

$$
f(z) g(z)=O\left(z^{\mu}\right) O\left(z^{\nu}\right)=O\left(z^{\mu+\nu}\right),
$$

and

$$
\begin{aligned}
|f(z) g(z)-f(\zeta) g(\zeta)| & \leqq|f(z)||g(z)-g(\zeta)|+|g(\zeta)||f(z)-f(\zeta)| \\
& \leqq K_{1}|z|^{\mu+\nu-\beta}|z-\zeta|^{\beta}+K_{2}|z|^{\mu+\nu-\alpha}|z-\zeta|^{\alpha} \\
& \leqq K_{3}|z|^{\mu+\nu-\alpha^{\prime}}|z-\zeta|^{\alpha^{\prime}}
\end{aligned}
$$

since

$$
|z-\zeta|^{\beta}=|z-\zeta|^{\beta-\alpha^{\prime}}|z-\zeta|^{\alpha^{\prime}} \leqq \delta^{\beta-\alpha^{\prime}}|z|^{\beta-\alpha^{\prime}}|z-\zeta|^{\alpha^{\prime}}
$$

and

$$
|z-\zeta|^{\alpha} \leqq \delta^{\alpha-\alpha^{\prime}}|z|^{\alpha-\alpha^{\prime}}|z-\zeta|^{\alpha^{\prime}} \text {. }
$$

The proof then follows easily from induction.

We now state three lemmas. The analogous theorems for the real case follow without difficulty.

Lemma 1. Let $\mu>0$ and let $f(z) \in W_{\mu}^{M+\alpha}(S)$. Suppose also that $|f(z)| \geqq \delta_{1}|z|^{\mu}, z \in S$, for some $\delta_{1}>0$. Let $S^{\prime}$ be the range of $S$ and suppose $g(z) \in W_{\nu}^{N+\beta}(S)$. Then

$$
h(z)=g(f(z)) \in W_{\mu \nu}^{M^{\prime}+\alpha \beta}(S)
$$

where $M^{\prime}=\min (M, N)$.

Proof. It is clear that $h(z)=O\left(z^{\mu \nu}\right)$. Next, for $|z-\zeta|<\delta|z|, \delta|\zeta|$

$$
\begin{aligned}
|h(z)-h(\zeta)| & =|g(f(z))-g(f(\zeta))| \\
& \leqq K_{1} \max \left(|f(z)|^{\nu-\beta},|f(\zeta)|^{\nu-\beta}\right)|f(z)-f(\zeta)|^{\beta} \\
& \leqq K_{2} \max \left(|z|^{\mu(\nu-\beta)},|\zeta|^{\mu(\nu-\beta)}\right)|z|^{(\mu-\alpha) \beta}|z-\zeta|^{\alpha \beta} \\
& \leqq K_{3}|z|^{\mu \nu-\alpha \beta}|z-\zeta|^{\alpha \beta}
\end{aligned}
$$


provided $|f(z)-f(\zeta)| \leqq \delta|f(z)|, \delta|f(\zeta)|$. In the contrary case, however, suppose $|f(z)| \leqq|f(\zeta)|$ and $|f(z)-f(\zeta)|>\delta|f(z)|$. Then

$$
\begin{aligned}
\frac{|g(f(z))-g(f(\zeta))|}{|z-\zeta|^{\alpha \beta}} & \leqq K_{4}|g(f(z))-g(f(\zeta))||z|^{(\mu-\alpha) \beta}|f(z)-f(\zeta)|^{-\beta} \\
& \leqq K_{5} \max \left(|f(z)|^{\nu},|f(\zeta)|^{\nu}\right)|f(z)|^{-\beta}|z|^{(\mu-\alpha) \beta} \\
& \leqq K_{6} \max \left(|z|^{\mu \nu},|\zeta|^{\mu \nu}\right)|z|^{-\alpha \beta} \\
& \leqq K_{7}|z|^{\mu \nu-\alpha \beta}
\end{aligned}
$$

and thus $h(z) \in W_{\mu \nu \nu}^{\alpha \beta}(S)$.

Writing $f=\varphi+i \psi, \varphi$, $\psi$ real, we have

$$
\frac{\partial}{\partial x} h(z)=g_{\varphi}(f(z)) \varphi_{x}(z)+g_{\psi}(f(z)) \psi_{x}(z) \text {. }
$$

Now by definition $g_{\varphi} \in W_{\nu-1}^{N-1+\beta}\left(S^{\prime}\right)$, and thus $g_{\varphi}(f(z)) \in W_{(\nu-1) \mu}^{M^{\prime}-1+\alpha \beta}(S)$ as well as $\varphi_{x}, \psi_{x} \in W_{\mu-1}^{M-1+\alpha}(S)$, and thus, by Proposition 6 ,

$$
\frac{\partial}{\partial x} h(z) \in W_{\mu \nu-1}^{\mu^{\prime}-1+\alpha \beta}(S) .
$$

The lemma follows by similar arguments.

Lemma 2. Let $f(z)$ map $S$ onto a set $S^{\prime}$ in such a way that $f(z)$ is conformal on the interior of $S$, and suppose $f(z) \in W_{\mu}^{M+\alpha}(S), \mu>0$. Assume also that $\left|f^{\prime}(z)\right| \geqq \delta_{1}|z|^{\mu-1}, z \in S$, for some $\delta_{1}>0$. Let $g(\zeta)$ be the function inverse to $f(z)$ which maps the interior of $S^{\prime}$ into $S$, and assume that $g(\zeta) \in C^{M+\alpha}\left(S^{\prime}\right)$ (this is the case if $S$ and $S^{\prime}$ have sufficiently smooth boundaries).

Then

$$
g(\zeta) \in W_{1 / \mu}^{M+\alpha}\left(S^{\prime}\right)
$$

Proof. Let $z_{0}$ be fixed. Then for $|z|$ sufficiently small we have $|f(z)| \leqq\left|f\left(z_{0}\right)\right|$. Thus

$$
\begin{aligned}
\left|f\left(z_{0}\right)\right|-|f(z)| & =|| f\left(z_{0}\right)|-| f(z)|| \leqq\left|f\left(z_{0}\right)-f(z)\right| \\
& =\left|\int_{z}^{z_{0}} f^{\prime}(w) d w\right| \\
& \leqq \int_{z}^{z_{0}} K_{1}|w|^{\mu-1}|d w| \leqq K_{2}\left(1+\left|z_{0}\right|^{\mu}-|z|^{\mu}\right)
\end{aligned}
$$

where the path of integration is taken to be a union of paths $\arg w$ : const. and $|w|=$ const. Thus

$$
|f(z)| \geqq K_{3}+K_{2}|z|^{\mu} \geqq K_{2}|z|^{\mu} .
$$

Since $|f(z)| \leqq K_{4}|z|^{\mu}$ we have 


$$
\left|g^{\prime}(\zeta)\right|=\left|f^{\prime}(z)\right|^{-1} \leqq \delta_{1}^{-1}|z|^{1-\mu} \leqq K_{5}|\zeta|^{1 / \mu-1} .
$$

By Propositions 2 and 3 we have $g(\zeta) \in W_{1 / \mu}^{a}\left(S^{\prime}\right)$.

Next,

$$
\begin{aligned}
\frac{\left|g^{\prime}\left(\zeta_{1}\right)-g^{\prime}\left(\zeta_{2}\right)\right|}{\left|\zeta_{1}-\zeta_{2}\right|^{\alpha}} & =\frac{\left|f^{\prime}\left(z_{2}\right)-f^{\prime}\left(z_{1}\right)\right|}{\left|f^{\prime}\left(z_{1}\right)\right|\left|f^{\prime}\left(z_{2}\right)\right|\left|z_{1}-z_{2}\right|^{\alpha}} \frac{\left|z_{1}-z_{2}\right|^{\alpha}}{\left|\zeta_{1}-\zeta_{2}\right|^{\alpha}} \\
& \leqq K_{6}\left|z_{1}\right|^{1-\mu}\left|z_{2}\right|^{1-\mu}\left|z_{1}\right|^{\mu-1-\alpha}\left|f^{\prime}(z)\right|^{-\alpha}
\end{aligned}
$$

where $z$ lies between $z_{1}$ and $z_{2}$. Since $1-\delta \leqq\left|z_{1} / z_{2}\right| \leqq 1+\delta$ we have

$$
\frac{\left|g^{\prime}\left(\zeta_{1}\right)-g^{\prime}\left(\zeta_{2}\right)\right|}{\left|\zeta_{1}-\zeta_{2}\right|^{\alpha}} \leqq K_{7}\left|z_{1}\right|^{1-\mu-\alpha-(\mu-1) \alpha} \leqq K_{8}\left|\zeta_{1}\right|^{(1 / \mu)-1-\alpha}
$$

Thus $g(z) \in W_{1 / \mu}^{1+\alpha}\left(S^{\prime}\right)$. The proof follows by induction.

Lemma 3. Let $f(z) \in W_{\mu}^{N+\alpha}(S)$ and let $P(z)$ be a polynomial of degree $<\mu$ with $P(0)=1$. Let $\gamma$ be a positive real number. Then there exists a function $f_{1}(z) \in W_{\mu}^{N+\alpha}(S)$ and a polynomial $P_{1}(z)$ of degree $<\mu$ such that

$$
(P(z)+f(z))^{\gamma}=P_{1}(z)+f_{1}(z) .
$$

Proof. The proof follows easily from the binomial theorem.

In obtaining the asymptotic expansions we shall come across certain integrals which were studied in [8], [5], and [12]. To estimate these integrals we use the following lemmas. The first was proved in [8] and [5]. The second is a generalization of a theorem in [8], [5], and [12] and will be proved in $\S 9$. The integrals are Lebesgue integrals extended over positive values of $t$. The variable $z$ lies on the logarithmic Riemann surface with branch point at the origin. The kernel of the integrands is the function $\log (t-z)$ which we define in the following way. For fixed $t$ we make cuts along the Riernann surface from $t e^{2 \pi i k}$ to $\infty e^{2 \pi i k}, k=0, \pm 1, \pm 2, \cdots$. The logarithm is uniquely defined, except for $z$ lying on these cuts, as the analytic continuation of the logarithm which is real for $0<|z|<t, \arg z=0$.

Lemma 4. Let $A$ be a positive number, $\mu$ a real number $>-1$, and $n$ a nonnegative integer. For $0<\arg z<2 \pi$, let

$$
f(z)=\int_{0}^{A} t^{\mu}(\log t)^{n} \log (t-z) d t
$$

Then there exists a polynomial $P(\log z)$ and a power series $p(z)$ which converges for $|z|<A$, such that 


$$
f(z)=z^{\mu+1} P(\log z)+p(z) .
$$

If $\mu$ is an integer the polynomial $P$ is of degree $n+1$; otherwise it is of degree $n$.

LEMMA 5. Let $\mu$ be a real number $>-1$ which is not an integer, and let $\beta(t) \in W_{\mu}^{N-1+\alpha}$ for $0<t \leqq A$. For $0<\arg z<\pi$, let

$$
g(z)=\int_{0}^{A} \beta(t) \log (t-z) d t .
$$

Then there exists a polynomial $q(z)$ of degree $<\mu+1$ such that

$$
\varphi(z) \equiv g(z)-q(z) \in W_{\mu+1}^{N+\alpha}((0, \pi)) .
$$

A similar result obtains for $-\pi<\arg z<0$, with the same polynomial $q(z)$.

5. Preliminary results. It follows from Warschawski [10] that $F^{-1}(z)$, which maps $D$ onto the upper half plane, satisfies the relation

$$
F^{-1}(z)=z^{1 / \tau} \varphi(z)
$$

where $\varphi(z)$ is continuous in $D \cup \Gamma_{1} \cup \Gamma_{2} \cup\{0\}$ and $\varphi(0) \neq 0$. We shall show in this section that $F^{-1}(z) \in W_{1 / \tau}^{N+\alpha}\left(D \cup \Gamma_{1} \cup \Gamma_{2}\right)$.

It follows easily from the Cauchy integral theorem that

$$
F^{-1}(z) \in W_{1 / \tau}^{N+\alpha}([\lambda, \pi \tau-\lambda])
$$

where $\lambda$ is a small positive number: one simply examines the integral

$$
\frac{d^{n}}{d z^{n}} F^{-1}(z)=\frac{n !}{2 \pi i} \oint \frac{F^{-1}(\zeta) d \zeta}{(\zeta-z)^{n+1}}
$$

taken over a circle about $z$ of radius $\delta|z|$, bearing in mind that $F^{-1}(z)=0\left(z^{1 / \tau}\right)$ as $z \rightarrow 0, z \in D \cup \Gamma_{1} \cup \Gamma_{2}$. Thus it will suffice to show that $F^{-1}(z) \in W_{1 / \tau}^{N+\alpha}\left(D^{\prime}\right)$ and $F^{-1}(z) \in W_{1 / \tau}^{N+\alpha}\left(D^{\prime \prime}\right)$ where $D^{\prime}=D \cap\{z: \arg z \geqq$ $\pi \tau-2 \lambda\}$ and $D^{\prime \prime}=D \cap\{z: \arg z \leqq 2 \lambda\}$. Because of the symmetry between $\Gamma_{1}$ and $\Gamma_{2}$ we need only show that $F^{-1}(z) \in W_{1 / \tau}^{N+\alpha}\left(D^{\prime}\right)$.

Next, if we have $V(z)=\operatorname{Im} F^{-1}(z) \in W_{1 / \tau}^{N+\alpha}\left(D^{\prime}\right)$, then, by Warschawski's result above and the Cauchy-Riemann equations, we have $\operatorname{Re} F^{-1}(z) \in W_{1 / \tau}^{N+\alpha}\left(D^{\prime}\right)$, and thus $F^{-1}(z) \in W_{1 / \tau}^{N+\alpha}\left(D^{\prime}\right)$. Thus we shall show $V(z) \in W_{1 / \tau}^{N+\alpha}\left(D^{\prime}\right)$.

Now we make a transformation which has the effect of straightening out $\Gamma_{2}$. Let $y=\beta(x)$ be a parametrization of $\Gamma_{2}$ (if $\tau=1 / 2$ or $3 / 2$ this is impossible; but a small rotation about the origin would take care of this difficulty). Then it can be shown that $\beta(x) \in C^{N+a}$ for $0 \leqq x \leqq A$, where $A$ is a small positive number; furthermore, by 
the hypotheses of $\S 2$, for $0 \leqq n \leqq N, 0 \leqq x_{1}, x_{2} \leqq x_{0}$

$$
\sup _{x_{1} \neq x_{2}} \frac{\left|\beta^{(n)}\left(x_{1}\right)-\beta^{(n)}\left(x_{2}\right)\right|}{\left|x_{1}-x_{2}\right|^{\alpha}}<\infty .
$$

We make the transformation $\xi=x, \eta=y-\beta(x)$, and set $v(\xi, \eta)=$ $V(x, y)$. Then $v$ is defined (at least) for $0<\xi^{2}+\eta^{2}<A_{1},-\xi \tan \delta \leqq$ $\eta<0$, provided $A_{1}$ and $\delta$ are chosen small enough. The points $(\xi, \eta)$ are images of a subset of the points $(x, y)$ such that $\left(\pi \tau-\delta_{1}\right) x \leqq y \leqq$ $\beta(x)$, where $\delta_{1}$ is a small positive number. Since $\beta(x)=O(x)$, we find that $\delta_{2} \leqq y / x \leqq 1 / \delta_{2}$ for some $\delta_{2}>0$, and thus, since

$$
\begin{aligned}
\xi^{2}+\eta^{2} & =x^{2}+y^{2}-2 y \beta(x)+(\beta(x))^{2}, \\
\delta_{3} & \leqq \frac{x^{2}+y^{2}}{\xi^{2}+\eta^{2}} \leqq 1 / \delta_{3}
\end{aligned}
$$

for some $\delta_{3}>0$. Since $V(x, y)=O\left(z^{1 / \tau}\right)$, we have $v(\xi, \eta)=O\left(\zeta^{1 / \tau}\right)$, where $\zeta=\xi+i \eta$.

We now state a lemma which is a special case of a theorem of Agmon, Douglis and Nirenberg ([1], pp. 657-660). Let $0<R<1$ and let $S$ be the semicircle $\xi^{2}+\eta^{2}<R, \eta \leqq 0$. For $\zeta \in S$ let $d_{\zeta}$ denote the distance from $\zeta$ to the circular part of the boundary of $S$.

Lemma 6. Let $u(\xi, \eta)$ be a solution of a uniformly elliptic partial differential equation

$$
L u=a u_{\xi \xi}+2 b u_{\xi \eta}+c u_{\eta \eta}+d u_{\xi}+e u_{\eta}+f u=0,
$$

whose coefficients are $C^{N-2+\alpha}$ in $S$ with uniform $\alpha$-Hölder constants. Let $u(\xi, 0)=0$ for $-R<\xi<R$. If $u \in C^{2+\alpha}(S)$ then $u \in C^{N+\alpha}(S)$, and there exists a constant $K$, independent of $u$ and $R$, such that

$$
|u|_{N+\alpha} \leqq K \sup _{\zeta \in S}|u(\zeta)|
$$

where

$$
\begin{aligned}
|u|_{N+\alpha}= & \sup _{4\left|\zeta_{1}-\zeta_{2}\right|<d} d_{\zeta_{1}, d \zeta_{2}}^{N+\infty} \frac{\left|D^{N} u\left(\zeta_{1}\right)-D^{N} u\left(\zeta_{2}\right)\right|}{\left|\zeta_{1}-\zeta_{2}\right|^{\alpha}} \\
& +\sum_{k=0}^{N} \sup _{\zeta \in S} d_{\zeta}^{k}\left|D^{k} u(\zeta)\right| ;
\end{aligned}
$$

the suprema are taken over all kth and Nth order derivatives of $u$.

Since $V(x, y)$ is harmonic, we have

$$
L v \equiv v_{\xi \xi}+\left(1+\beta^{\prime}(\xi)^{2}\right) v_{\eta \eta}-2 \beta^{\prime}(\xi) v_{\xi \eta}-\beta^{\prime \prime}(\xi) v_{\eta}=0
$$

for $0<\xi^{2}+\eta^{2}<A_{1},-\xi \tan \delta \leqq \eta \leqq 0$. Also 


$$
v(\xi, 0)=V(x, \beta(x))=0 .
$$

We now apply the lemma to $v$ and the semicircles

$$
\left(\xi-\xi_{0}\right)^{2}+\eta^{2} \leqq \xi_{0}^{2} \sin ^{2} \delta, \eta \leqq 0
$$

where $0<\xi_{0}<(1 / 2) A_{1}$; these semicircles are tangent to the rays $\eta=0$, and $\eta=-\xi \tan \delta$. In each semicircle we have, for some $K_{1}>0$,

$$
\sup |v(\zeta)| \leqq K_{1}|\zeta|^{1 / \tau} \text {. }
$$

In the semicircle $\left(\xi-\xi_{0}\right)^{2}+\eta^{2} \leqq\left((1 / 2) \xi_{0} \sin \delta\right)^{2}, \eta \leqq 0$, we have $d_{\zeta} \geqq$ $(1 / 2) \xi_{0} \sin \delta$. Thus for $\left(\xi-\xi_{0}\right)^{2}+\eta^{2} \leqq\left((1 / 2) \xi_{0} \sin \delta\right)^{2}, \eta \leqq 0$, we have

$$
\begin{aligned}
\zeta^{k}\left|D^{k} v(\zeta)\right| & \leqq\left(\xi_{0}\left(1+\frac{1}{2} \sin \delta\right)\right)^{k}\left|D^{k} v(\zeta)\right| \\
& \leqq\left(\frac{2}{\sin \delta}\left(1+\frac{1}{2} \sin \delta\right) d_{\zeta}\right)^{k}\left|D^{k} v(\zeta)\right| \leqq K_{2}|\zeta|^{1 / \tau}
\end{aligned}
$$

for $0 \leqq k \leqq N$. Thus $v(\zeta) \in W_{1 / \tau}^{N}\left(\left[-\delta_{4}, 0\right]\right)$ where $\delta_{4}$ is small. By the mean value theorem $v(\zeta) \in W_{1 / \tau}^{N-1+\alpha}\left(\left[-\delta_{4}, 0\right]\right)$. To estimate $\left|D^{N} v\left(\zeta_{1}\right)-D^{N} v\left(\zeta_{2}\right)\right|$ we use the lemma again; the details are similar to those above. Thus we can conclude that $v(\zeta) \in W_{1 / \tau}^{N+\alpha}\left(\left[-\delta_{4}, 0\right]\right)$. Since

$$
\delta_{3} \leqq \frac{x^{2}+y^{2}}{\xi^{2}+\eta^{2}} \leqq \frac{1}{\delta_{3}},
$$

it follows, by easy calculations, that for some small positive $\lambda$,

$$
V(z) \in W_{1 / \tau}^{N+\alpha}([\pi \tau-\lambda, \pi \tau]) \text {. }
$$

Thus we conclude that $F^{-1}(z) \in W_{1 / \tau}^{N+\alpha}\left(D \cup \Gamma_{1} \cup \Gamma_{2}\right)$.

6. A preliminary transformation. From now on for the sake of definiteness we will assume that $\Gamma_{1}$ is tangent to the positive $x$ axis at the origin and that $\Gamma_{2}$ is tangent to the $\operatorname{ray} \arg z=\pi \tau$ at the origin.

We set $H(z)=(F(z))^{1 / \tau}$. Then $H(z)$ maps the upper half plane conformally onto a domain $D^{\prime}$ which is the image of $D$ under the transformation $z \rightarrow z^{1 / \tau}$. $\quad D^{\prime}$ is partially bounded by curves $\Gamma_{1}^{\prime}$ and $\Gamma_{2}^{\prime}$ which have horizontal tangents at the origin. From the binomial theorem it is clear that theorem 1 is equivalent to an asymptotic expansion

$$
\begin{aligned}
& H(z)=z P\left(z, z^{\tau}\right)+o\left(z^{(N-1) \tau-\varepsilon}\right) \quad(\alpha \text { irrational }) \\
& H(z)=z P\left(z, z^{\tau}, z^{p} \log z\right)+o\left(z^{(N-1) \tau-\varepsilon}\right) \quad(\alpha=p / q)
\end{aligned}
$$

as $z \rightarrow 0,0 \leqq \arg z \leqq \pi$, where $\varepsilon>0$ can be chosen arbitrarily small 
and the polynomial $P$ has a nonvanishing constant term; furthermore we must show that we can differentiate (6.1) $N$ times. Since Theorems 2,3 and 4 follow directly from Theorem 1, we need only prove (6.1).

By Lemma 1, and since $F^{-1}(z) \in W_{1 / \tau}^{N+\alpha}\left(D \cup \Gamma_{1} \cup \Gamma_{2}\right)$, we have

$$
H^{-1}(z)=F^{-1}\left(z^{\tau}\right) \in W_{1}^{N+\alpha^{2}}\left(D^{\prime} \cup \Gamma_{1}^{\prime} \cup \Gamma_{2}^{\prime}\right) \text {. }
$$

By Lemma 2, $H(z) \in W_{1}^{N+\alpha^{2}}([0, \pi])$.

7. An integral representation. We will now construct an integral representation for $H(z)$ based on the equations for $\Gamma_{1}$ and $\Gamma_{2}$. Let $F(z)=\xi+i \eta$. Then we have

$$
\eta=\sum_{n=1}^{N-1} c_{n} \xi^{n}+\varphi_{1}(\xi)
$$

where $\varphi_{1} \in W_{N}^{N+\alpha}$; this is merely the Taylor series for $\Gamma_{1}$, and is valid for $0 \leqq \xi \leqq \xi_{0}$.

We will now adopt the convention of dropping subscripts on coefficients whose value is unimportant; then we have

$$
\eta=\sum_{n=1}^{N-1} c \xi^{n}+\varphi_{1}(\xi) \text {. }
$$

With $w=H(z)=u+i v$, we have

$$
\begin{aligned}
w=(\xi+i \eta)^{1 / \tau} & =\xi^{1 / \tau}\left(1+i \sum_{n=1}^{N} c \xi^{n-1}+\frac{\varphi_{1}(\xi)}{\xi}\right)^{1 / \tau} \\
& =\xi^{1 / \tau}\left(a+\sum_{n=1}^{N} c \xi^{n}+\varphi_{2}(\xi)\right),
\end{aligned}
$$

and by Lemma $3, \varphi_{2} \in W_{N-1}^{N+\alpha}$. It is readily seen that $R e a \neq 0$. Then we have, by separating real and imaginary parts,

$$
\begin{aligned}
& u=a_{1} \xi^{1 / \tau}\left(1+c \xi+c \xi^{2}+\cdots+c \xi^{N-2}+\varphi_{3}(\xi)\right) \\
& v=\xi^{1 / \tau}\left(c+c \xi+c \xi^{2}+\cdots+c \xi^{N-2}+\varphi_{4}(\xi)\right)
\end{aligned}
$$

with $\varphi_{3}, \varphi_{4} \in W_{N-1}^{N+\alpha}$. Next,

$$
u^{\tau}=a_{1}^{\tau} \xi\left(1+c \xi+c \xi^{2}+\cdots+c \xi^{N-2}+\varphi_{5}(\xi)\right)
$$

with $\varphi_{5} \in W_{N-1}^{N+\alpha}$. As $a_{1}^{\tau} \neq 0$, we have, by the inverse function theorem,

$$
\xi=u^{\tau}\left(c+c u^{\tau}+c u^{2 \tau}+\cdots+c u^{(N-2) \tau}+\varphi_{6}\left(u^{\tau}\right)\right)
$$

where $\varphi_{6}$, considered as a function of $u^{\tau}$, belongs to $W_{N-1}^{N+\alpha}$. Thus by Lemma 1, $\varphi_{7}(u)=\varphi_{6}\left(u^{\tau}\right) \in W_{(N-1) \tau}^{N+\alpha^{2}}$. Substituting (7.3) in the right side of (7.2), we obtain 


$$
\begin{aligned}
v= & u\left(\sum_{j=0}^{N-2} c u^{j \tau}+\varphi_{7}(u)\right)^{1 / \tau} \\
& \times\left(\sum_{j=0}^{N-2} c u^{j \tau}\left(\sum_{k=0}^{N-2} c u^{k \tau}+\varphi_{7}(u)\right)^{j}+\varphi_{4}(\xi)\right) .
\end{aligned}
$$

We set

$$
\varphi_{8}(u)=\varphi_{4}(\xi)=\varphi_{4}\left(u^{\tau}\left(\sum_{j=0}^{N-2} c u^{j \tau}+\rho_{7}(u)\right)\right) .
$$

It is easily checked that $\xi(u) \in W_{\tau}^{N+\alpha^{2}}$ as a function of $u$, and thus $\varphi_{8}(u) \in W_{(N-1) \tau}^{N+\alpha^{2}}$. Thus, expanding the right side of (7.4), it follows that

$$
v=u\left(c+c u^{\tau}+\cdots+c u^{(N-1) \tau}+\varphi_{9}(u)\right)
$$

with $\varphi_{9} \in W_{(N-1) \tau}^{N+\alpha^{2}}$. Finally, $\left.(d v / d u)\right|_{u=0}=0$, and thus

$$
v=u\left(c u^{\tau}+c u^{2 \tau}+\cdots+c u^{(N-1) \tau}+\varphi_{9}(u)\right) .
$$

This equation is valid for $v$ and $u$ defined on the segment $y=0$, $0 \leqq x \leqq A$, provided $A$ is chosen small enough.

If $0<\tau<1 / 2$ or $3 / 2<\tau \leqq 2$ we can repeat the same argument on $\Gamma_{2}$ : note that we never used the fact that $\Gamma_{1}$ has a horizontal tangent, but only that $\Gamma_{1}^{\prime}$ (and $\Gamma_{2}^{\prime}$ ) has a horizontal tangent at the origin. If $1 / 2<\tau<3 / 2$, we replace $\xi$ by $|\xi|$; and for $0<\tau \leqq 2$, we replace $u$ by $|u|$.

Finally, if $\tau=1 / 2$ or $3 / 2$ we begin with the equation

$$
\xi=\sum_{n-1}^{N} c \eta^{n}+\varphi_{10}(\eta)
$$

and carry through with the roles of $\xi$ and $\eta$ reversed. Thus we have, for $-A \leqq x \leqq 0, y=0$,

$$
v=u\left(c u^{\tau}+c u^{2 \tau}+\cdots+c u^{(N-1) \tau}+\varphi_{11}(u)\right)
$$

with $\varphi_{11} \in W_{(N-1) \tau}^{N+\alpha^{2}}$.

We now consider the Green's function for the upper half plane

$$
G(t, z)=-\frac{1}{2 \pi}\{\log |t-z|+\log |t-\bar{z}|\},
$$

where $t=x_{t}+i y_{t}$. It is easily seen that $\left(\partial / \partial y_{t}\right) G\left(x_{t}, z\right)=0$. We apply Green's theorem to the functions $G(t, z)$ and $u(t)=R e H(t)$ on the semi-circle $0<|t|<A, y_{t}>0$, and obtain

$$
u(z)=\int_{-A}^{A} G(t, z) \frac{\partial}{\partial y_{t}} u(t) d t+\int_{\substack{y_{t}>0 \\|t|=A}}\left(u G_{n_{t}}-G u_{n_{t}}\right) d s_{t}
$$


where $s_{t}$ represents arc length and $n_{t}$ the outward normal. By (7.7) we have

$$
\int_{\substack{y_{t}>0 \\|t|=A}}\left(u G_{n_{t}}-G u_{n_{t}}\right) d s_{t}=p(z)+p(\bar{z})
$$

where $p(z)$ is a power series which converges for $|z|<A$. Also, for $y_{t}=0$,

$$
\begin{aligned}
G(t, z) & =-\frac{1}{2 \pi}\{\log |t-z|+\log |t-\bar{z}|\} \\
& =-\frac{1}{2 \pi} \log |t-z|^{2} \\
& \equiv-\frac{1}{2 \pi}\{\log (t-z)+\log (t-\bar{z})\}
\end{aligned}
$$

Here we define $\log (t-z)$ as the analytic continuation of the logarithm which is real for $0<|z|<t, \arg z=0$. The congruence holds modulo $2 \pi i$; however, each of the logarithms on the right side has imaginary part $>-\pi$ and $<\pi$. Thus we may replace the congruence by equality. With these observations in mind, we obtain

$$
\begin{aligned}
u(z)= & -\frac{1}{2 \pi} \int_{-A}^{A} \frac{\partial}{\partial y_{t}} u(t) \\
& \times\{\log (t-z)+\log (t-\bar{z})\} d t+p(z)+p(\bar{z}) .
\end{aligned}
$$

Since $u(z)=\operatorname{Re} H(z)$ and $p(z)$ has real coefficients, we replace (7.8) by the equation of which it is the real part, namely

$$
H(z)=-\frac{1}{\pi} \int_{-\Lambda}^{A} \frac{\partial}{\partial y_{t}} u(t) \log (t-z) d t+p(z)+\text { const. , }
$$

where the constant takes care of the nonuniqueness of the conjugate harmonic function of $u(z)$. We now drop this constant, changing $p(z)$ if necessary, and use (7.5) and (7.6), together with

$$
\frac{\partial}{\partial y_{t}} u(t)=-\frac{\partial}{\partial x_{t}} v(t)
$$

to obtain

$$
\begin{aligned}
H(z)= & \frac{1}{\pi} \int_{-A}^{0} u_{t}(t, 0)\left\{\sum_{j=1}^{N-1} c u^{j \tau}+\varphi(u)\right\} \log (t-z) d t \\
& +\frac{1}{\pi} \int_{0}^{A} u_{t}(t, 0)\left\{\sum_{j=1}^{N-1} c u^{j \tau}+\psi(u)\right\} \log (t-z) d t+p(z) .
\end{aligned}
$$

Here 


$$
\varphi(u)=\frac{d}{d u}\left(u \varphi_{11}(u)\right) \in W_{(N-1) \tau}^{N-1+\alpha^{2}}
$$

and

$$
\psi(u)=\frac{d}{d u}\left(u \varphi_{9}(u)\right) \in W_{(N-1) \tau}^{N-1+\alpha^{2}}
$$

Furthermore, (7.9) is valid for $0 \leqq \arg z \leqq \pi, 0<|z|<A$.

8. Obtaining the asymptotic expansions. We have, for $-A \leqq$ $t<0, H(t) \in W_{1}^{N+\alpha^{2}}$ and thus $u(t) \in W_{1}^{N+\alpha^{2}}$. Hence

$$
\begin{aligned}
(u(t))^{n \tau} & \in W_{n \tau}^{N+\alpha^{3}} \\
u_{t} & \in W_{0}^{N-1+\alpha^{2}}
\end{aligned}
$$

and thus

$$
u_{t}(t, 0)\left\{\sum_{j=1}^{N-1} c u^{j \tau}+\varphi(u)\right\} \in W_{\tau}^{N-1+\alpha^{3}}
$$

as a function of $t,-A \leqq t<0$. Similarly

$$
u_{t}(t, 0)\left\{\sum_{j=1}^{N-1} c u^{j \tau}+\psi(u)\right\} \in W_{\tau}^{N-1+\alpha^{3}}
$$

for $0<t \leqq A$. Thus by Lemma, 5 , if $\tau \neq 1,2$,

$$
H(z)=a z+b z^{2}+\chi_{1}(z)
$$

where $\chi_{1}(z) \in W_{1+\tau}^{N+\alpha^{3}}((0, \pi))$. As $H(z)$ has $\alpha$-Hölder continuous $N$ th derivatives for $0 \leqq \arg z \leqq \pi$, we must have $\chi_{1}(z) \in W_{1+\tau}^{N+\alpha^{3}}([0, \pi])$.

If $\tau=1$ or 2 Lemma 5 will not apply. However, if $\varepsilon$ is any small positive number we can replace the $W_{\tau}^{N-1+\alpha^{3}}$ of (8.1) and (8.2) with $W_{\tau-c}^{N-1+\alpha^{3}}$, and thus we can always write

$$
H(z)=a z+b z^{2}+\chi_{1}(z)
$$

where $\chi_{1}(z) \in W_{1+\tau-\varepsilon}^{N+x^{3}}([0, \pi])$.

We now prove Theorem 1 by induction. In the future we shall use the symbol $\alpha$ to represent any number between 0 and 1 , and $\varepsilon$ to represent an arbitrarily small positive number such that $n \tau-\varepsilon$ is not an integer for $0 \leqq n \leqq N$. In particular we write $\chi_{1}(z) \in W_{1+\tau-\varepsilon}^{N+\alpha}([0, \pi])$.

First let $\alpha$ be irrational. Assume that for some $m$, with $0<m<$ $N-1$, that

$$
H(z)=z P_{m}\left(z, z^{\tau}\right)+\chi_{m}(z)
$$

where $P_{m}\left(z, z^{\tau}\right)$ is a polynomial in its arguments such that $P_{m}(0,0) \neq 0$ and $\chi_{m}(z) \in W_{1+m \tau-\varepsilon}^{N+a}([0, \pi])$. That this is the case for $m=1$ follows 
from the fact that the constant $a$ of (8.3) is not equal to zero; this follows from (5.1) and the definition of $H(z)$.

Then by the inductive hypothesis we have, for $-A \leqq t<0$,

$$
u(t, 0)=t\left(\sum_{k+l \tau<m \tau} c_{k l} t^{k+l \tau}+\varphi_{12}(t)\right)
$$

with $\varphi_{12}(t) \in W_{m \tau-\varepsilon}^{N+\alpha}$ and $c_{00} \neq 0$. A similar equation holds for $0<t \leqq A$. Then

$$
u^{n \tau}=t^{n \tau}\left\{\sum_{k+l \tau<m \tau} c t^{k+l \tau}+\varphi_{13}^{n}(t)\right\}
$$

with $\varphi_{13}^{n} \in W_{m \tau-\varepsilon}^{N+a}$, and

$$
u_{t}(t, 0)=\sum_{k+l \tau<m \tau} c(k+1+l \tau) t^{k+l \tau}+\varphi_{14}(t)
$$

with $\varphi_{14} \in W_{m \tau-\varepsilon}^{N-1+\alpha}$. Also, since $\varphi(u) \in W_{(N-1) \tau}^{N+\alpha}$ as a function of $u$, $\varphi(u(t)) \in W_{(N-1) \tau}^{N-1+\alpha}$ as a function of $t$. Thus, cross-multiplying, collecting terms, and using Lemmas 1; 2 and 3, we obtain

$$
u_{t}(t, 0)\left\{\sum_{\jmath=1}^{N-1} c u^{j \tau}+\varphi(u)\right\}=\sum_{k+l \tau<m \tau} c t^{k+l \tau}+\varphi_{14}(t)+\varphi_{15}(t)
$$

with $\varphi_{14}(t) \in W_{(m+1) \tau-\varepsilon}^{N-1+\alpha}$ and $\varphi_{15} \in W_{(N-1) \tau-\varepsilon}^{N-1+\alpha}$. By the inductive hypothesis $m+1 \leqq N-1$ and we may write $\varphi_{16}=\varphi_{14}+\varphi_{15} \in W_{(m+1) \tau-\varepsilon}^{N-1+\alpha}$. Clearly a similar equation holds for $0<t \leqq A$, and, applying Lemmas 4 and 5 we obtain

$$
H(z)=\sum c_{k l} z^{k+l \tau}+\chi_{m+1}(z)
$$

with $\chi_{m+1}(z) \in W_{(m+1) \tau+1-\varepsilon}^{N+\infty}((0, \pi))$. As $H$ has continuous $N$ th derivatives, $\chi_{m+1} \in W_{(m+1) \tau+1-\varepsilon}^{N+\alpha}([0, \pi])$. By Warschawski's results $c_{10} \neq 0$. Finally, setting $m=N-2$, and $\chi_{N-1}(z)=o\left(z^{(N-1) \tau+1-2 \varepsilon}\right)$, we have, with $2 \varepsilon$ replaced by $\varepsilon$,

$$
H(z)=z P_{N-1}\left(z, z^{\tau}\right)+o\left(z^{(N-1) \tau+1-\varepsilon}\right)
$$

as $z \rightarrow 0,0 \leqq \arg z \leqq \pi$, and, for $0 \leqq n \leqq N$

$$
\frac{d^{n}}{d z^{n}}\left(H(z)-z P_{N-1}\left(z, z^{\tau}\right)\right)=o\left(z^{(N-1) \tau+1-n-\varepsilon}\right)
$$

as $z \rightarrow 0,0 \leqq \arg z \leqq \pi$.

Now let $\tau=p / q$, a reduced fraction. For $0<m<N-1$ we assume that

$$
H(z)=z P_{m}\left(z, z^{\tau}, z^{p} \log z\right)+\chi_{m}(z)
$$

with $\chi_{m}(z) \in W_{1+m \tau-\varepsilon}^{N+a}([0, \pi])$, and $P_{m}(0,0,0) \neq 0$. Then, for $-A \leqq t \leqq 0$, 


$$
\begin{aligned}
u(t, 0) & =t \cdot \sum c t^{j+k \tau}\left(t^{p} \log t\right)^{l}+\varphi_{17}(t) \\
u_{t}(t, 0) & =\sum c t^{j+k \tau}\left(t^{p} \log t\right)^{l}+\varphi_{18}(t) \\
u^{n \tau} & =t^{n \tau}\left\{\sum c t^{j+k \tau}\left(t^{p} \log t\right)^{l}+\varphi_{19}(t)\right\}
\end{aligned}
$$

where $\varphi_{17}, \varphi_{19} \in W_{1+m \tau-\varepsilon}^{N+a}$ and $\varphi_{18} \in W_{m \tau-\varepsilon}^{N-1+\infty}$. Thus

$$
u_{t}(t, 0)\left\{\sum_{\jmath=1}^{N-1} c u^{j \tau}+\varphi(u(t))\right\}=\sum c t^{j+k \tau}(\log t)^{l}+\varphi_{20}(t)
$$

where $j \geqq 0,1 \leqq k \leqq q, 0 \leqq l \leqq j / p, j+k \tau<(m+1) \tau$ and $\varphi_{20} \in W_{(m \neq 1) \tau}^{N-1+\alpha}$. A similar equation obtains for $0<t \leqq A$. Applying Lemmas 4 and 5 we obtain

$$
H(z)=\sum a_{j_{k l}} z^{j+1+k \tau}(\log z)^{l^{\prime}}+\chi_{m+1}(z)
$$

with $\chi_{m+1} \in W_{(m+1) \tau+1-\varepsilon}^{N+a}([0, \pi])$. Terms of the form $t^{j+k \tau}(\log t)^{l}$, with $k<q$, contribute terms of the form $z^{j+1+k \tau}(\log z)^{l^{\prime}}$ with $l^{\prime} \leqq l<l+1$. With $k=q$, however, higher powers of the logarithm appear, and we must then show $j+1+k \tau \geqq l^{\prime}+1$, where $l^{\prime} \leqq l+1$. But then

$$
\begin{aligned}
j+1+k \tau & =j+1+p \\
& \geqq p l+1+p \geqq p(l+1)+1 \geqq l+2 \geqq l^{\prime}+1 .
\end{aligned}
$$

Thus we can write

$$
H(z)=z P_{m+1}\left(z, z^{\tau}, z^{p} \log z\right)+\chi_{m+!}(z),
$$

and, for $m=N-2$ and $0 \leqq n \leqq N$,

$$
\frac{d^{n}}{d z^{n}} H(z)=\frac{d^{n}}{d z^{n}}\left(z P_{N--1}\left(z, z^{\tau}, z^{p} \log z\right)\right)+o\left(z^{(N-1) \tau+1-n-\varepsilon}\right)
$$

as $z \rightarrow 0,0 \leqq \arg z \leqq \pi$.

9. Proof of Lemma 5. Suppose that $q(z)$ exists and $\varphi(z)=$ $g(z)-q(z) \in W_{\mu+1}^{N}((0, \pi])$. Then it follows that $\varphi(z) \in W_{\mu+1}^{N-1+\alpha}((0, \pi])$. Hence we need only show that there exists a polynomial $q(z)$ such that

$$
\varphi^{(N)}(z) \equiv g^{(N)}(z)-q^{(N)}(z) \in W_{\mu-N+1}^{\alpha}((0, \pi]) .
$$

We break the proof into three parts, numbered I, II and III.

I. First we assume $0<\arg z \leqq \delta$. We have

$$
g^{(N)}(z)=-\int_{0}^{A}(N-1) ! \frac{\beta(t)}{(t-z)^{N}} d t .
$$

We write, with $r=|z|$, 


$$
\int_{0}^{A}=\int_{0}^{x-r / 2}+\int_{x-r / 2}^{x+r / 2}+\int_{x+r / 2}^{A}=I_{1}+I_{2}+I_{3}
$$

Throughout the proof we shall use constants $C_{1}, C_{2}, C_{3}, \cdots$, which are independent of $z$; to simplify notation we shall use one symbol $C$ to denote all such constants. $I_{1}$ is bounded in absolute value by

$$
C \int_{0}^{x-r / 2} t^{\mu}(\delta x)^{-N} d t \leqq C r^{\mu+1-N},
$$

where we have used $|\beta(t)| \leqq C t^{\mu}$

For $I_{2}$ we expand $\beta(t)$ in a Taylor series about the point $x$ and get

$$
\begin{aligned}
I_{2}=\sum_{k=0}^{N-2} \frac{(N-1) !}{k !} \beta^{(k)}(x) & \int_{x-r / 2}^{x+r / 2} \frac{(t-x)^{k}}{(t-z)^{N}} d t \\
& +\int_{x-r / 2}^{x+r / 2} \frac{\beta^{(N-1)}(\tau)(t-x)^{N-1}}{(t-z)^{N}} d t
\end{aligned}
$$

where $\tau$ lies between $x$ and $t$.

The integral term $J_{k}$ arising from the $k$ th term of (9.1) can be written in the form

$$
\begin{aligned}
J_{k} & =C \beta^{(k)}(x) \int_{-r / 2}^{r / 2} \frac{t^{k}}{(t-i y)^{N}} d t \\
& =C \sum_{j=0}^{k}\left(\begin{array}{c}
k \\
j
\end{array}\right) \beta^{(k)}(x) \int_{-r / 2}^{r / 2} \frac{(t-i y)^{j}(i y)^{k-j}}{(t-i y)^{N}} d t \\
& =\sum_{j=0}^{k} C_{j} \beta^{(k)}(x)(i y)^{k-j}\left[\left(\frac{r}{2}-i y\right)^{j-N+1}-\left(-\frac{r}{2}-i y\right)^{j-N+1}\right],
\end{aligned}
$$

and thus, since $\left|\beta^{(k)}(x)\right| \leqq C x^{\mu-k}$,

$$
\left|J_{k}\right| \leqq C \sum_{j=0}^{k} x^{\mu-k} y^{k-j} 2\left(\frac{r}{2}\right)^{j-N+1} \leqq C r^{\mu-N+1} .
$$

The last integral on the right side of (9.1) we write in the form

$$
\begin{aligned}
\int_{-r / 2}^{r / 2} & \frac{\beta^{(N-1)}\left(\tau_{1}+x\right) t^{N-1}}{(t-i y)^{N}} d t \\
& =\int_{0}^{r / 2} t^{N-1}\left\{\frac{\beta^{(N-1)}\left(\tau_{1}+x\right)}{(t-i y)^{N}}-\frac{\beta^{(N-1)}\left(\tau_{2}+x\right)}{(t+i y)^{N}}\right\} d t
\end{aligned}
$$

where $0<\tau_{1},-\tau_{2}<t$. We write the term in brackets in two parts, and get

$$
\int_{0}^{r / 2} t^{N-1}\left\{\frac{\beta^{(N-1)}\left(\tau_{1}+x\right)}{(t-i y)^{N}}-\frac{\beta^{(N-1)}\left(\tau_{1}+x\right)}{(t+i y)^{N}}\right\} d t
$$




$$
+\int_{0}^{r / 2} t^{N-1}\left\{\frac{\beta^{(N-1)}\left(\tau_{1}+x\right)}{(t+i y)^{N}}-\frac{\beta^{(N-1)}\left(\tau_{2}+x\right)}{(t+i y)^{N}}\right\} d t .
$$

The first integral is equal to

$$
\begin{aligned}
2 i \operatorname{Im} & \int_{0}^{r / 2} t^{N-1} \frac{\beta^{(N-1)}\left(\tau_{1}+x\right)}{(t-i y)^{N}} d t \\
& =2 i \operatorname{Im} \int_{0}^{r / 2} \frac{t^{N-1}(t+i y)^{N} \beta^{(N-1)}\left(\tau_{1}+x\right) d t}{\left(t^{2}+y^{2}\right)^{N}} .
\end{aligned}
$$

We make the change of variables $t=s y$ to obtain

$$
\begin{aligned}
2 i \operatorname{Im} & \int_{0}^{r / 2 y} \frac{s^{N-1}(s+i)^{N} \beta^{(N-1)}(\sigma+x) d s}{\left(s^{2}+1\right)^{N}} \\
& =2 i \operatorname{Im} \sum_{k=1}^{N}\left(\begin{array}{l}
N \\
k
\end{array}\right) \int_{0}^{r / 2 y} \frac{s^{2 N-1-k} i^{k} \beta^{(N-1)}(\sigma+x) d s}{\left(s^{2}+1\right)^{N}}
\end{aligned}
$$

where $\sigma<r / 2$; this is bounded in absolute value by

$$
C \sum_{k=1}^{N} \int_{0}^{\infty} \frac{s^{2 N-1-k}}{\left(s^{2}+1\right)^{N}}|r / 2 \pm x|^{\mu-N+1} d s \leqq C r^{\mu-N+1} .
$$

The second integral on the right side of (9.2) is bounded absolutely by

$$
C \int_{0}^{r / 2}|2 t|^{\alpha}|x \pm r / 2|^{\mu-N+1-\alpha} t^{-1} d t \leqq C r^{\mu+1-N}
$$

To handle $I_{3}$ we observe that

$$
\frac{1}{(t-z)^{N}}=\sum_{k=0}^{\infty}(k+\underset{k}{N}-1) z^{k} t^{-N-k} .
$$

Let $m$ be the integer such that $\mu<m<\mu+1$ and assume $m \geqq N$. We have

$$
\begin{aligned}
I_{3}= & \sum_{k=0}^{m-N} \frac{(k+N-1) !}{k !} z^{k} \int_{0}^{A} \beta(t) t^{-N-k} d t \\
& -\sum_{k=0}^{m-N} \frac{(k+N-1) !}{k !} z^{k} \int_{0}^{x+r / 2} \beta(t) t^{-N-k} d t \\
& +\int_{x+r / 2}^{A} \sum_{k=m-N+1}^{\infty} \frac{(k+N-1) !}{k !} z^{k} \beta(t) t^{-N-k} d t .
\end{aligned}
$$

We set

$$
q_{1}(z) \equiv-\sum_{k=0}^{m-N} \frac{(k+N-1) !}{k !} z^{k} \int_{0}^{A} \beta(t) t^{-N-k} d t
$$

If $m<N$ we set $q_{1}(z) \equiv 0$, and the last sum of (9.3) begins with 
$k=0$. In any event, $q(z)$ will be taken such that $q^{(N)}(z) \equiv q_{1}(z)$; its exact form is given in [12].

Thus to prove that $\varphi^{(N)}(z) \equiv g^{(N)}(z)-q_{1}(z) \in W_{\mu-N+1}((0, \delta))$, we need only estimate the last two terms on the right side of (9.3). We have

$$
\begin{aligned}
& \left|\sum_{k=0}^{m-N} \frac{(k+N-1) !}{k !} z^{k} \int_{0}^{x+r / 2} \beta(t) t^{-N-k} d t\right| \\
& \leqq C \sum_{k=0}^{m-N} r^{k} \int_{0}^{x+r / 2} t^{\mu-N-k} d t=C r^{\mu+1-N},
\end{aligned}
$$

and

$$
\begin{aligned}
& \left|\int_{x+r / 2}^{A} \sum_{k=m-N+1}^{\infty} \frac{(k+N-1) !}{k !} z^{k} \beta(t) t^{-N-k} d t\right| \\
& \quad \leqq C \sum_{k=m-N+1}^{\infty} \frac{(k+N-1) !}{k !} r^{k} \int_{x+r / 2}^{\infty} t^{\mu-N-k} d t \\
& \quad \leqq C \sum_{k=m-N+1}^{\infty} \frac{(k+N-1) !}{k !} \frac{r^{\mu+1-N}}{|\mu-N-k+1|}\left(\cos \delta+\frac{1}{2}\right)^{\mu-N-k+1} \\
& \quad=C r^{\mu+1-N}
\end{aligned}
$$

where we assume $\cos \delta>1 / 2$. Thus $\varphi^{(N)}(z) \in W_{\mu-N+1}((0, \delta))$.

II. For $\delta \leqq \arg z \leqq \pi$, observe that $\varphi(z)$ is analytic for $|z|>0$. That $\varphi(z) \in W_{\mu+1}$ follows from [12]. By Cauchy's theorem

$$
\varphi^{(N)}(z)=\frac{N !}{2 \pi i} \int \frac{\varphi(\zeta) d \zeta}{(\zeta-z)^{N+1}}
$$

where the integral is taken around a circle with $z$ as center and radius $\delta_{1}|z|$, where $\delta_{1}$ is a small positive number. Then

$$
\left|\varphi^{(N)}(z)\right| \leqq C\left(2 \pi \delta_{1}|z|\right)\left(|z|\left(1 \pm \delta_{1}\right)\right)^{\mu+1}\left(\delta_{1}|z|\right)^{-N-1}=C r^{\mu+1-N} .
$$

III. We will now show that

$$
\left|\varphi^{(N)}(z)-\varphi^{(N)}(\zeta)\right| \leqq C|z|^{\mu+1-N-\alpha}|z-\zeta|^{\alpha},
$$

for $|z-\zeta| \leqq \delta|z|, \delta|\zeta|$. First, this inequality follows immediately from (9.4) for $\delta \leqq \arg z, \arg \zeta \leqq \pi$. Thus we will restrict ourselves to the range $0<\arg z, \arg \zeta \leqq \delta$. We have

$$
\begin{aligned}
\varphi^{(N)}(z)-\varphi^{(N)}(\zeta)= & -\int_{0}^{x-r / 2}(N-1) ! \beta(t)\left\{\frac{1}{(t-z)^{N}}-\frac{1}{(t-\zeta)^{N}}\right\} d t \\
& -\int_{x-r / 2}^{x+r / 2}(N-1) ! \beta(t)\left\{\frac{1}{(t-z)^{N}}-\frac{1}{(t-\zeta)^{N}}\right\} d t \\
& +\sum_{k=0}^{m-N} \frac{(k+N-1) !}{k !}\left(z^{k}-\zeta^{k}\right) \int_{0}^{x+r / 2} \beta(t) t^{-N-k} d t
\end{aligned}
$$




$$
\begin{aligned}
& -\int_{x+r / 2}^{A} \sum_{\substack{k=m-N+1 \\
k \geqq 0}}^{\infty} \frac{(k+N-1) !}{k !}\left(z^{k}-\zeta^{k}\right) \beta(t) t^{-N-k} d t \\
= & I^{1}+I^{2}+I^{3}+I^{4} .
\end{aligned}
$$

Then

$$
I^{1}=-(N-1) ! \int_{0}^{x-r / 2} \beta(t) \sum_{k=1}^{N} \frac{t^{N-1}(-1)^{k}\left[\zeta^{k}-z^{k}\right] d t}{(t-z)^{N}(t-\zeta)^{N}}
$$

Since $|\zeta-z| \leqq \delta|z|$ and $|\zeta| \leqq(1+\delta)|z|$, we have

$$
\begin{aligned}
\left|\zeta^{k}-z^{k}\right| & \leqq|\zeta-z|^{\alpha}|\zeta-z|^{1-\alpha} \sum_{j=0}^{k-1}|\zeta|^{k-j-1}|z|^{j} \\
& \leqq C|z|^{k-\alpha}|\zeta-z|^{\alpha} .
\end{aligned}
$$

Hence

$$
\begin{aligned}
\left|I^{1}\right| & \leqq C \sum_{k=1}^{N}|z-\zeta|^{\alpha}|z|^{k-\alpha} \int_{0}^{x-r / 2} t^{\mu+N-k}\left(\frac{r}{2}\right)^{-N}\left(r\left(\frac{1}{2}-\delta\right)\right)^{-N} d t \\
& \leqq C|z-\zeta|^{\alpha}|z|^{\mu-N+1-\alpha} .
\end{aligned}
$$

Similarly, we can assume the sum in $I_{3}$ begins with $k=1$, and we obtain

$$
\begin{aligned}
\left|I^{3}\right| & \leqq C|z-\zeta|^{\alpha} \sum_{k=1}^{m-N}|z|^{k-\alpha} \int_{0}^{x+r / 2} t^{\mu-N-k} d t \\
& \leqq C|z-\zeta|^{\alpha}|z|^{\mu-N+1-\alpha} .
\end{aligned}
$$

Likewise

$$
\begin{aligned}
\left|I^{4}\right| & \leqq C|z-\zeta|^{\alpha} \sum_{k=m-N+1}^{\infty} \frac{(k+N-1) !}{k>0}|z|^{k-\alpha} \int_{x+r / 2}^{\infty} t^{\mu-N-1 k} d t \\
& \leqq C|z-\zeta|^{\alpha} \sum_{\substack{k=m-N+1 \\
k>0}}^{\infty} \frac{(k+N-1) !}{k !}|z|^{\mu-N+1-\alpha} \frac{\left(\cos \delta+\frac{1}{2}\right)^{\mu-N-k+1}}{|\mu-N-k+1|} \\
& =C|z|^{\mu-N+1-\alpha}|z-\zeta|^{\alpha} .
\end{aligned}
$$

Finally we must evaluate $I^{2}$. We write

$$
\beta(t)=\sum_{k=0}^{N-2} \frac{\beta^{(k)}(x)}{k !}(t-x)^{k}+\psi(x, t)
$$

where

$$
\psi(x, t)=\int_{x}^{t} \frac{(t-\sigma)^{N-2}}{(N-2) !} \beta^{(N-1)}(\sigma) d \sigma
$$




$$
\beta(t)=\sum_{k=0}^{N-2} \frac{\beta^{(k)}(\xi)}{k !}(t-\xi)^{k}+\psi(\xi, t) .
$$

Then we have

$$
\begin{aligned}
I^{2}= & -\sum_{k=0}^{N-2} \frac{(N-1) !}{k !} \int_{x-r / 2}^{x+r / 2}\left\{\frac{\beta^{(k)}(x)(t-x)^{k}}{(t-z)^{N}}-\frac{\beta^{(k)}(\xi)(t-\xi)^{k}}{(t-\zeta)^{N}}\right\} d t \\
& -(N-1) \int_{x-r / 2}^{x+r / 2}\left\{\frac{\psi(x, t)}{(t-z)^{N}}-\frac{\psi(\xi, t)}{(t-\zeta)^{N}}\right\} d t \\
= & \sum_{k=0}^{N-2} J^{k}+J^{N-1} .
\end{aligned}
$$

With

$$
(t-x)^{k}=\sum_{j=0}^{k}\left(\begin{array}{c}
k \\
j
\end{array}\right)(t-z)^{j}(z-x)^{k-j}
$$

we have, for $k \leqq N-2$,

$$
\begin{aligned}
J^{k}= & -\frac{(N-1) !}{k !} \sum_{j=0}^{k}\left(\begin{array}{c}
k \\
j
\end{array}\right) \\
& \times \int_{x-r / 2}^{x+r / 2}\left[\beta^{(k)}(x)(t-z)^{j-N}(z-x)^{k-j}-\beta^{(k)}(\xi)(t-\zeta)^{j-N}(\zeta-x)^{k-j}\right] d t \\
= & -\frac{(N-1) !}{k !} \sum_{j=0}^{k}\left(\begin{array}{l}
k \\
j
\end{array}\right) \frac{1}{j-N+1} \\
& \times\left\{\beta^{(k)}(x)(z-x)^{k-j}\left(\left(x+\frac{r}{2}-z\right)^{j-N+1}-\left(x-\frac{r}{2}-z\right)^{j-N+1}\right)\right. \\
& \left.\quad-\beta^{(k)}(\xi)(\zeta-\xi)^{k-j}\left(\left(x+\frac{r}{2}-\zeta\right)^{j-N+1}-\left(x-\frac{r}{2}-\zeta\right)^{j-N+1}\right)\right\}
\end{aligned}
$$

To the term in brackets we add and subtract

$$
\beta^{(k)}(x)(\zeta-\xi)^{k-j}\left(\left(x+\frac{r}{2}-\zeta\right)^{j-N+1}-\left(x-\frac{r}{2}-\zeta\right)^{j-N+1}\right) .
$$

Then the $j$ th bracketed term becomes

$$
\begin{aligned}
\beta^{(k)}(x) & {\left[(z-x)^{k-j}\left(\left(x+\frac{r}{2}-z\right)^{j-N+1}-\left(x-\frac{r}{2}-z\right)^{j-N+1}\right)\right.} \\
& \left.-(\zeta-\xi)^{k-j}\left(\left(x+\frac{r}{2}-\zeta\right)^{j-N+1}-\left(x-\frac{r}{2}-\zeta\right)^{j-N+1}\right)\right] \\
+ & \left(\beta^{(k)}(x)-\beta^{(k)}(\xi)\right) \\
& \times\left[(\zeta-\xi)^{k-j}\left(\left(x+\frac{r}{2}-\zeta\right)^{j-N+1}-\left(x-\frac{r}{2}-\zeta\right)^{j-N+1}\right)\right] .
\end{aligned}
$$

Thus to evaluate $J^{k}, k \leqq N-2$, it suffices to evaluate each term of (9.5). 
Since $|\zeta-\xi|=|\eta| \leqq|\zeta| \leqq|z|(1+\delta)$, and

$$
\begin{aligned}
& \left|x+\frac{r}{2}-\zeta\right| \geqq r\left(\cos \delta-\delta-\frac{1}{2}\right) \\
& \left|x-\frac{r}{2}-\zeta\right| \geqq r\left(\frac{1}{2}-\delta\right),
\end{aligned}
$$

the second term of (9.5) is bounded absolutely by

$$
C x^{\mu-k-\alpha}|x-\xi|^{\alpha} r^{k-N+1} \leqq C r^{\mu-N+1-\alpha}|z-\zeta|^{\alpha} .
$$

To evaluate the first term of (9.5), consider the function

$$
f(p, q)=(i q)^{k-j}\left(\left(x+\frac{r}{2}-p-i q\right)^{j-N+1}-\left(x-\frac{r}{2}-p-i q\right)^{j-N+1}\right)
$$

and its first partial derivatives, $f_{1}(p, q)=f_{p}(p, q)$, and $f_{2}(p, q)=f_{q}(p, q)$. Then we must evaluate

$$
\beta^{(k)}(x)(f(x, y)-f(\xi, \eta)) .
$$

By the mean value theorem we have, for some $\lambda$ with $0<\lambda<1$,

$$
\begin{aligned}
f(x, y)-f(\xi, \eta)= & (x-\xi) f_{1}(x+\lambda(\xi-x), y+\lambda(\eta-y)) \\
& +(y-\eta) f_{2}(x+\lambda(\xi-x), y+\lambda(\eta-y)) .
\end{aligned}
$$

Then using previously mentioned inequalities for $|\zeta-\xi|,|x \pm r / 2-\zeta|$, etc., it follows easily that for $k \leqq N-2$

$$
\left|J^{k}\right| \leqq C r^{\mu-N+1-\alpha}|z-\zeta|^{\alpha} .
$$

Thus to finish the proof we must evaluate

$$
\begin{aligned}
J^{N-1}=-(N-1) & \int_{x-r / 2}^{x+r / 2}\left\{\frac{\psi(x, t)}{(t-z)^{N}}-\frac{\psi(\xi, t)}{(t-\zeta)^{N}}\right\} d t \\
=-(N-1) & \int_{-r / 2}^{r / 2} \frac{\psi(x, x+t)}{(t-i y)^{N}} \\
& +(N-1) \int_{x-\xi-r / 2}^{x-\xi+r / 2} \frac{\psi(\xi, \xi+t)}{(t-i \eta)^{N}} d t .
\end{aligned}
$$

We will assume $x \geqq \xi$; were $x<\xi$ another similar argument would prevail. Then

$$
\begin{aligned}
J^{N-1}=(N-1) \int_{-r / 2}^{r / 2} & \left\{\frac{\psi(\xi, \xi+t)}{(t-i \eta)^{N}}-\frac{\psi(x, x+t)}{(t-i y)^{N}}\right\} d t \\
-(N-1) \int_{-r / 2}^{x-\xi-r / 2} \frac{\psi(\xi, \xi+t)}{(t-i \eta)^{N}} d t & +(N-1) \int_{r / 2}^{x-\xi+r / 2} \frac{\psi(\xi, \xi+t)}{(t-i \eta)^{N}} d t .
\end{aligned}
$$


By definition, for $t \geqq 0$,

$$
|\psi(\xi, \xi+t)| \leqq \int_{\xi}^{\xi+t} \frac{(\xi+t-\sigma)^{N-2}}{(N-2) !}\left|\beta^{(N-1)}(\sigma)\right| d \sigma \leqq C \xi^{\mu-N+1} t^{N-1},
$$

and thus the third integral on the right side of (9.6) is bounded absolutely by

$$
\begin{aligned}
C|z|^{\mu-N+1} \int_{r / 2}^{x-\xi+r / 2} t^{-1} d t & \leqq C|z|^{\mu-N+1}|x+\xi|\left(\frac{r}{2}\right)^{-1} \\
& \leqq C|z-\zeta|^{\alpha}|z|^{\mu-N+1-\alpha} .
\end{aligned}
$$

We handle the second integral of (9.6) in the same fashion.

Thus we have left to evaluate

$$
\begin{aligned}
\int_{-r / 2}^{r / 2} & \left\{\frac{\psi(\xi, \xi+t)}{(t-i \eta)^{N}}-\frac{\psi(x, x+t)}{(t-i y)^{N}}\right\} d t \\
= & \int_{0}^{r / 2}\left\{\frac{\psi(\xi, \xi+t)}{(t-i \eta)^{N}}+(-1)^{N} \frac{\psi(\xi, \xi-t)}{(t+i \eta)^{N}}\right. \\
& \left.\quad-\frac{\psi(x, x+t)}{(t-i y)^{N}}-(-1)^{N} \frac{\psi(x, x-t)}{(t+i y)^{N}}\right\} d t \\
= & \int_{0}^{r / 2} d t \int_{0}^{t} \frac{(t-s)^{N-2}}{(N-2) !}\left\{\frac{\beta^{(N-1)}(\xi+s)}{(t-i \eta)^{N}}-\frac{\beta^{(N-1)}(\xi-s)}{(t+i \eta)^{N}}\right. \\
& \left.\quad-\frac{\beta^{(N-1)}(x+s)}{(t-i y)^{N}}+\frac{\beta^{(N-1)}(x-s)}{(t+i y)^{N}}\right\} d s
\end{aligned}
$$

where we have recalled the definition of $\psi$.

We write

$$
\begin{aligned}
& \beta^{(N-1)}(\xi+s)=\beta^{(N-1)}(\xi)+K_{1}(\xi, s) s^{\alpha} \\
& \beta^{(N-1)}(\xi-s)=\beta^{(N-1)}(\xi)+K_{2}(\xi, s) s^{\alpha} \\
& \beta^{(N-1)}(x+s)=\beta^{(N-1)}(x)+K_{3}(x, s) s^{\alpha} \\
& \beta^{(N-1)}(x-s)=\beta^{(N-1)}(x)+K_{4}(x, s) s^{\alpha}
\end{aligned}
$$

where $\left|K_{i}(s)\right| \leqq C|z|^{\mu-N+1-\alpha}$. Also

$$
s^{\alpha}\left|K_{1}(\xi, s)-K_{3}(x, s)\right| \leqq C|z|^{\mu-N+1-\alpha}|z-\zeta|^{\alpha}
$$

and

$$
s^{\alpha}\left|K_{1}(\xi, s)-K_{3}(x, s)\right| \leqq C|z|^{\mu-N+1-\alpha} s^{\alpha} .
$$

Similar inequalities hold for $s^{\alpha}\left|K_{2}-K_{4}\right|$.

Next, (9.7) becomes 


$$
\begin{aligned}
& \int_{0}^{r / 2} d t \int_{0}^{t} \frac{(t-s)^{N-2}}{(N-2) !}\left\{\beta^{(N-1)}(\xi)\left(\frac{1}{(t-i \eta)^{N}}-\frac{1}{(t+i \eta)^{N}}\right)\right. \\
&-\left.\beta^{(N-1)}(x)\left(\frac{1}{(t-i y)^{N}}-\frac{1}{(t+i y)^{N}}\right)\right\} d s \\
&+\int_{0}^{r / 2} d t \int_{0}^{t} \frac{(t-s)^{N-2}}{(N-1) !}\left\{\frac{K_{1}(\xi, s) s^{\alpha}}{(t-i \eta)^{N}}\right. \\
&\left.-\frac{K_{2}(\xi, s) s^{\alpha}}{(t+i \eta)^{N}}-\frac{K_{3}(x, s) s^{\alpha}}{(t-i y)^{N}}+\frac{K_{4}(x, s) s^{\alpha}}{(t+i y)^{N}}\right\} d s .
\end{aligned}
$$

Notice that

$$
\begin{aligned}
& \int_{0}^{r / 2} d t \int_{0}^{t} \frac{(t-s)^{N-2}}{(N-2) !}\left(\frac{1}{(t-i \eta)^{N}}-\frac{1}{(t+i \eta)^{N}}\right) d s \\
&= \int_{0}^{r / 2} \frac{t^{N-1}}{(N-1) !}\left(\frac{1}{(t-i \eta)^{N}}-\frac{1}{(t+i \eta)^{N}}\right) d t \\
&=\sum_{k=0}^{N-1}\left(\begin{array}{c}
N-1 \\
k
\end{array}\right) \frac{1}{(N-1) !} \quad \times \int_{0}^{r / 2}\left\{(t-i \eta)^{-1-k}(i \eta)^{k}-(t+i \eta)^{-1-k}(-i \eta)^{k}\right\} d t \\
&=\sum_{k=0}^{N-1} C_{k}\left\{\left(\frac{r}{2}-i \eta\right)^{-k}(i \eta)^{k}-\left(\frac{r}{2}+i \eta\right)^{-k}(-i \eta)^{k}\right\} .
\end{aligned}
$$

Thus the first term of (9.9) is bounded absolutely by terms of the form

$$
\begin{aligned}
& \beta^{(N-1)}(\xi)\left\{\left(\frac{i \eta}{r / 2-i \eta}\right)^{k}-\left(\frac{-i \eta}{r / 2+i \eta}\right)^{k}\right\} \\
& -\beta^{(N-1)}(x)\left\{\left(\frac{i y}{r / 2-i y}\right)^{k}-\left(\frac{-i y}{r / 2+i y}\right)^{k}\right\} \\
& =2 i \beta^{(N-1)}(\xi) \operatorname{Im}\left[\left(\frac{i \eta}{r / 2-i \eta}\right)^{k}-\left(\frac{i y}{r / 2-i y}\right)^{k}\right] \\
& +2 i \operatorname{Im}\left[\left(\frac{i y}{r / 2-i y}\right)^{k}\right]\left(\beta^{(N-1)}(\xi)-\beta^{(N-1)}(x)\right) \text {. }
\end{aligned}
$$

The second term is bounded by $C|z|^{\mu-N+1-\alpha}|z-\zeta|^{\alpha}$. The first, using the mean value theorem, is bounded by

$$
C|z|^{\mu-N+1}|y-\eta|\left|\operatorname{Im} \frac{\sigma^{k-1} r i^{k}}{(r / 2-i \sigma)^{k+1}}\right|
$$

where $\sigma$ lies between $y$ and $\eta_{\text {. }}$ But then $\sigma \leqq C r,|r / 2-i \sigma| \leqq r / 2$, and hence $(9.10)$ is $\leqq$

$$
C|z|^{\mu-N+1}|y-\eta|^{\alpha} r^{1-\alpha} r^{-1} \leqq C|z|^{\mu-N+1-\alpha}|z-\zeta|^{\alpha} .
$$

Thus we are left with the second term of (9.9). By symmetry we need only consider 


$$
\begin{aligned}
& \int_{0}^{r / 2} d t \int_{0}^{t}(t-s)^{N-2} s^{\alpha}\left\{\frac{K_{1}(\xi, s)}{(t-i \eta)^{N}}-\frac{K_{3}(x, s) s^{\alpha}}{(t-i y)^{N}}\right\} d s \\
& =\int_{0}^{r / 2} d t \int_{0}^{t}(t-s)^{N-2} s^{\alpha}\left(K_{1}(\xi, s)-K_{3}(x, s)\right)(t-i \eta)^{-N} d s \\
& \quad+\int_{0}^{r / 2} d t \int_{0}^{t}(t-s)^{N-2} s^{\alpha} K_{3}(x, s)\left(\frac{1}{(t-i \eta)^{N}}-\frac{1}{(t-i y)^{N}}\right) d s .
\end{aligned}
$$

By (9.8), for any $\varepsilon>0$ we have

$$
s^{\alpha}\left|K_{1}(\xi, s)-K_{3}(x, s)\right| \leqq C_{1}^{\varepsilon} C_{1}^{1-\varepsilon}|z|^{\mu-N+1-\alpha} s^{\alpha \varepsilon}|x-\xi|^{\alpha(1-\varepsilon)} .
$$

Thus the first term on the right side of (9.11) is bounded in absolute value by

$$
\begin{gathered}
C C_{1}^{\varepsilon} C_{2}^{1-\varepsilon}|z-\zeta|^{\alpha(1-\varepsilon)}|z|^{\mu-N+1-\alpha} \int_{0}^{r / 2} d t \int_{0}^{t} t^{N-2} s^{\alpha \varepsilon}|t-i \eta|^{-N} d s \\
\leqq C C_{1}^{\varepsilon} C_{2}^{1-\varepsilon}|z-\zeta|^{\alpha(1-\varepsilon)}|z|^{\mu-N+1-\alpha} \int_{0}^{r / 2} t^{\alpha \varepsilon-1} d t \\
=C C_{1}^{\varepsilon} C_{2}^{1-\varepsilon}|z-\zeta|^{\alpha(1-\varepsilon)}|z|^{\mu-N+1-\alpha+\alpha \varepsilon} .
\end{gathered}
$$

Now let $\varepsilon \rightarrow 0$.

Finally, to evaluate the second term on the right side of (9.11), we observe that

$$
\begin{aligned}
& \left|\frac{1}{(t-i \eta)^{N}}-\frac{1}{(t-i y)^{N}}\right| \\
& \quad \leqq \sum_{k=1}^{N}\left(\begin{array}{l}
N \\
k
\end{array}\right) t^{N-k}\left|y^{k}-\eta^{k}\right||t-i \eta|^{-N}|t-i y|^{-N} \\
& \quad \leqq|z-\zeta|^{\alpha} \sum_{k=1}^{N} t^{N-k} y^{k-\alpha}|t-i \eta|^{-N}|t-i y|^{-N}
\end{aligned}
$$

where we have assumed that $y \geqq \eta$. Then

$$
\begin{aligned}
& \left|\int_{0}^{r / 2} d t \int_{0}^{t}(t-s)^{N-2} s^{\alpha} K_{3}(x, s)\left((t-i \eta)^{-N}-(t-i y)^{-N}\right) d s\right| \\
& \quad \leqq \sum_{k=1}^{N} C_{k} \int_{0}^{r / 2} t^{2 N-k-1+\alpha}|z|^{\mu-N+1-\alpha}|z-\zeta|^{\alpha} y^{k-\alpha}|t-i \eta|^{-N}|t-i y|^{-N} d t \\
& \quad \leqq C|z|^{\mu-N+1-\alpha}|z-\zeta|^{\alpha} \sum_{k=1}^{N} \int_{0}^{r / 2} t^{N-k-1+\alpha} y^{k-\alpha}|t-i y|^{-N} d t .
\end{aligned}
$$

With the change of variables $t=\tau y$, the $k$ th integral becomes

$$
\int_{0}^{r / 2 y} \tau^{N-k-1+\alpha}|\tau-i|^{-N} d t \leqq \int_{0}^{\infty} \tau^{N-k-1+\alpha}|\tau-i|^{-N} d t=C
$$

since $1 \leqq k \leqq N$. This completes the evaluation of $J^{N-1}$ and the theorem is proved. 


\section{REFERENCES}

1. S. Agmon, A. Douglis, L. Nirenberg, Estimates near the boundary for solutions of elliptic partial differential equations satisfying general boundary conditions. I, Comm. Pure Appl. Math. 12 (1959), 623-727.

2. N. Bourbaki, Fonctions D'Une Variable Reelle, vol. XII, no. 1132, pp. 83.

3. D. S. Carter, Local behavior of plane gravity flows at the confluence of free boundaries and analytic fixed boundaries, J. Math. Mech. 10 (1961), 441-450.

4. O. D. Kellogg, Harmonic functions and Green's integral, Trans. Amer. Math. Soc, 13 (1912), 109-132.

5. R. S. Lehman, Developments in the neighborhood of the beach of surface waves over an inclined bottom, Comm. Pure Appl. Math. 7 (1954), 393-439.

6. - Development of the mapping function at an analytic corner, Pacific J. Math. 7 (1957), 1437-1449.

7. Developments at an analytic corner of solutions of elliptic partial differential equations, J. Math. Mech. 8 (1959), 727-760.

8. Hans Lewy, Developments at the confluence of analytic boundary condition, Univ. of Calif. Publ. in Math. 1, no. 7 (1950), 247-280; also Proc. Int. Cong. Math. (1950), 601-605. 9. L. Lichtenstein, Über die konforme Abbildung ebener analytischer Gebiete mit Ecken, J. Reine Angew. Math. 140 (1911), 100-119.

10. S. Warschawski, Über das Verhalten der Ableitung der Abbildungsfunktion bei konformer Abbildung, Math A. 35 (1932), 321-456.

11. W. Wasow, Asymptotic development of the solution of Dirichlet's problem at analytic corners, Duke Math. J. 24 (1957), 47-56.

12. N. W. Wigley, Asymptotic expansions at a corner of solutions of mixed boundary value problems, J. Math. Mech. 13 (1964) 549-576.

UNIVESITY OF ARIZONA 



\title{
PACIFIC JOURNAL OF MATHEMATICS
}

\author{
EDITORS
}

\author{
H. SAmelson \\ Stanford University \\ Stanford, California \\ R. M. Blumenthal \\ University of Washington \\ Seattle, Washington 98105
}

\author{
J. DugundjI \\ University of Southern California \\ Los Angeles, California 90007
}

*Richard Arens

University of California

Los Angeles, California 90024

\section{ASSOCIATE EDITORS}
E. F. BECKENBACH
B. H. NeUmanN
F. WOLF
K. YoSIDA

\section{SUPPORTING INSTITUTIONS}

\author{
UNIVERSITY OF BRITISH COLUMBIA \\ CALIFORNIA INSTITUTE OF TECHNOLOGY \\ UNIVERSITY OF CALIFORNIA \\ MONTANA STATE UNIVERSITY \\ UNIVERSITY OF NEVADA \\ NEW MEXICO STATE UNIVERSITY \\ OREGON STATE UNIVERSITY \\ UNIVERSITY OF OREGON \\ OSAKA UNIVERSITY \\ UNIVERSITY OF SOUTHERN CALIFORNIA
}

\author{
STANFORD UNIVERSITY \\ UNIVERSITY OF TOKYO \\ UNIVERSITY OF UTAH \\ WASHINGTON STATE UNIVERSITY \\ UNIVERSITY OF WASHINGTON \\ * * * * \\ AMERICAN MATHEMATICAL SOCIETY \\ CALIFORNIA RESEARCH CORPORATION \\ SPACE TECHNOLOGY LABORATORIES \\ NAVAL ORDNANCE TEST STATION
}

Mathematical papers intended for publication in the Pacific Journal of Mathematics should by typewritten (double spaced). The first paragraph or two must be capable of being used separately as a synopsis of the entire paper. It should not contain references to the bibliography. No separate author's resumé is required. Manuscripts may be sent to any one of the four editors. All other communications to the editors should be addressed to the managing editor, Richard Arens, at the University of California, Los Angeles, California 90024.

50 reprints per author of each article are furnished free of charge; additional copies may be obtained at cost in multiples of 50 .

The Pacific Journal of Mathematics is published quarterly, in March, June, September, and December. Effective with Volume 13 the price per volume (4 numbers) is $\$ 18.00$; single issues, $\$ 5.00$. Special price for current issues to individual faculty members of supporting institutions and to individual members of the American Mathematical Society: $\$ 8.00$ per volume; single issues $\$ 2.50$. Back numbers are available.

Subscriptions, orders for back numbers, and changes of address should be sent to Pacific Journal of Mathematics, 103 Highland Boulevard, Berkeley 8, California.

Printed at Kokusai Bunken Insatsusha (International Academic Printing Co., Ltd.), No. 6, 2-chome, Fujimi-cho, Chiyoda-ku, Tokyo, Japan.

PUBLISHED BY PACIFIC JOURNAL OF MATHEMATICS, A NON-PROFIT CORPORATION

The Supporting Institutions listed above contribute to the cost of publication of this Journal, but they are not owners or publishers and have no responsibility for its content or policies.

* Basil Gordon, Acting Managing Editor until February 1, 1966. 


\section{Pacific Journal of Mathematics}

\section{Vol. 15, No. $4 \quad$ December, 1965}

Robert James Blattner, Group extension representations and the structure space ........... 1101

Glen Eugene Bredon, On the continuous image of a singular chain complex .............. 1115

David Hilding Carlson, On real eigenvalues of complex matrices .................... 1119

Hsin Chu, Fixed points in a transformation group ............................. 1131

Howard Benton Curtis, Jr., The uniformizing function for certain simply connected Riemann

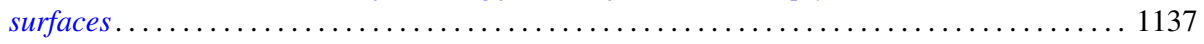

George Wesley Day, Free complete extensions of Boolean algebras................... 1145

Edward George Effros, The Borel space of von Neumann algebras on a separable Hilbert

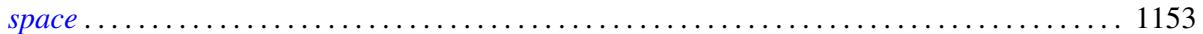

Michel Mendès France, $A$ set of nonnormal numbers ......................... 1165

Jack L. Goldberg, Polynomials orthogonal over a denumerable set ................ 1171

Frederick Paul Greenleaf, Norm decreasing homomorphisms of group algebras . . . . . . . . 1187

Fletcher Gross, The 2-length of a finite solvable group ........................ 1221

Kenneth Myron Hoffman and Arlan Bruce Ramsay, Algebras of bounded sequences ........ 1239

James Patrick Jans, Some aspects of torsion . . . . . . . . . . . . . . . . . . . . . . . 1249

Laura Ketchum Kodama, Boundary measures of analytic differentials and uniform

approximation on a Riemann surface ............................... 1261

Alan G. Konheim and Benjamin Weiss, Functions which operate on characteristic

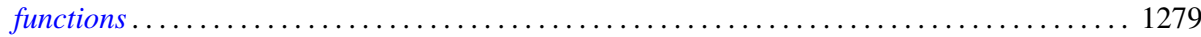

Ronald John Larsen, Almost invariant measures ............................ 1295

You-Feng Lin, Generalized character semigroups: The Schwarz decomposition ............ 1307

Justin Thomas Lloyd, Representations of lattice-ordered groups having a basis . . . . . . . . 1313

Thomas Graham McLaughlin, On relative coimmunity ....................... 1319

Mitsuru Nakai, $\Phi$-bounded harmonic functions and classification of Riemann surfaces ....... 1329

L. G. Novoa, On n-ordered sets and order completeness ..................... 1337

Fredos Papangelou, Some considerations on convergence in abelian lattice-groups . . . . . . . 1347

Frank Albert Raymond, Some remarks on the coefficients used in the theory of homology

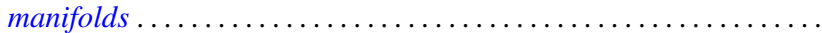

John R. Ringrose, On sub-algebras of a $C^{*}$-algebra .

Jack Max Robertson, Some topological properties of certain spaces of differentiable

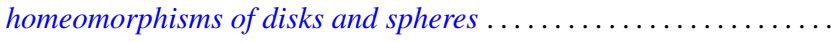

Zalman Rubinstein, Some results in the location of zeros of polynomials

Arthur Argyle Sagle, On simple algebras obtained from homogeneous general Lie triple systems. . . .

Hans Samelson, On small maps of manifolds ............................... 1401

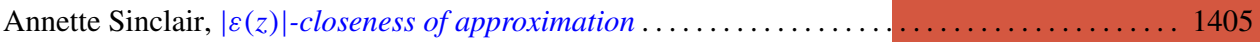

Edsel Ford Stiel, Isometric immersions of manifolds of nonnegative constant sectional curvature

Earl J. Taft, Invariant splitting in Jordan and alternative algebras ................. 1421

L. E. Ward, On a conjecture of R. J. Koch . . . . . . . . . . . . . . . . . . . . . . . . . . . 1429

Neil Marchand Wigley, Development of the mapping function at a corner . . . . . . . . . . 1435

Horace C. Wiser, Embedding a circle of trees in the plane ....................... 1463

Adil Mohamed Yaqub, Ring-logics and residue class rings . . . . . . . . . . . . . . . . 1465

John W. Lamperti and Patrick Colonel Suppes, Correction to: Chains of infinite order and their application to learning theory ........................................ 1471

Charles Vernon Coffman, Correction to: Non-linear differential equations on cones in Banach

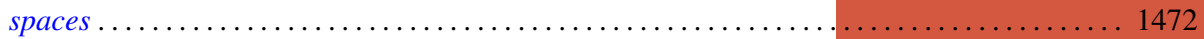

P. H. Doyle, III, Correction to: A sufficient condition that an $\operatorname{arc}$ in $S^{n}$ be cellular. . . . . . . . 1474

P. P. Saworotnow, Correction to: On continuity of multiplication in a complemented algebra 